\title{
Miniature Implantable Antennas for Biomedical Telemetry: From Simulation to Realization
}

\author{
Asimina Kiourti*, Student Member, IEEE, Jorge R. Costa, Senior Member, IEEE, \\ Carlos A. Fernandes, Senior Member, IEEE, André G. Santiago, and Konstantina S. Nikita, Senior Member, IEEE
}

\begin{abstract}
5 Abstract-We address numerical versus experimental design and testing of miniature implantable antennas for biomedical telemetry in the medical implant communications service band $(402-405 \mathrm{MHz})$. A model of a novel miniature antenna is initially proposed for skin implantation, which includes varying parameters to deal with fabrication-specific details. An iterative designand-testing methodology is further suggested to determine the parameter values that minimize deviations between numerical and experimental results. To assist in vitro testing, a low-cost technique is proposed for reliably measuring the electric properties of liquids without requiring commercial equipment. Validation is performed within a specific prototype fabrication/testing approach for miniature antennas. To speed up design while providing an antenna for generic skin implantation, investigations are performed inside a canonical skin-tissue model. Resonance, radiation, and safety performance of the proposed antenna is finally evaluated inside an anatomical head model. This study provides valuable insight into the design of implantable antennas, assessing the significance of fabrication-specific details in numerical simulations and uncertainties in experimental testing for miniature structures. The proposed methodology can be applied to optimize antennas for several fabrication/testing approaches and biotelemetry applications.
\end{abstract}

Index Terms-Biomedical telemetry, dielectric measurements, implantable antenna, in vitro, medical implant communications service (MICS) band.

Manuscript received February 14, 2012; revised April 5, 2012; accepted May 25, 2012. Date of publication; date of current version. This work was supported in part by the Fundação para a Ciência e Tecnologia, Portugal, under Project RFID Local PTDC/EEA-TEL/102390/2008. The work of A.K. was supported by the IEEE Antennas and Propagation Society Doctoral Research Award. Asterisk indicates corresponding author.

${ }^{*}$ A. Kiourti is with the School of Electrical and Computer Engineering and the Institute of Communications and Computer Systems, National Technical University of Athens, Athens 15780, Greece (e-mail: akiourti@biosim.ntua.gr).

J. R. Costa is with the Instituto de Telecomunicações and the Departamento de Ciências e Tecnologias da Informação, Instituto Universitário de Lisboa (ISCTE-IUL), Lisboa 1649-026, Portugal (e-mail: Jorge.Costa@1x.it.pt).

C. A. Fernandes is with Instituto de Telecomunicações and the Instituto Superior Técnico, Technical University of Lisbon, Lisboa 1049-001, Portugal (e-mail: carlos.fernandes@1x.it.pt).

A. G. Santiago is with Instituto Superior Técnico, Technical University of Lisbon, Lisboa 1049-001, Portugal (e-mail: andre.santiago.1988@gmail.com).

K. S. Nikita is with the School of Electrical and Computer Engineering and the Institute of Communications and Computer Systems, National Technical University of Athens, Athens 15780, Greece (e-mail: knikita@ece.ntua.gr).

Color versions of one or more of the figures in this paper are available online at http://ieeexplore.ieee.org.

Digital Object Identifier 10.1109/TBME.2012.2202659 equipment is most commonly performed in the medical implant communications service (MICS) band (402-405 MHz), which is regulated by the United States Federal Communications Commission [6] and the European Radiocommunications Committee [7]. Patch designs are preferred for implant-integrated antennas because of their flexibility in design, conformability, and shape [8].

Design of implantable patch antennas operating in the lowfrequency MICS band draws high-scientific interest to deal with miniaturization. The aim is to decrease the antenna ph $\equiv 1$ size, whil $\equiv$ reasing its electrical size. Rectangular 10 $\overline{240}$ - and $5760-\bar{\Omega}_{m}^{3}$ chest-implantable antennas have been reported [1], which use the high-permittivity $\left(\varepsilon_{r}=10.2\right)$ Rogers 3210 dielectric and apply a spiral radiator for size reduction. Addition of a shorting pin, thus, conversion to a planar inverted-F antenna (PIFA) acts like a ground plane on a monopole, and has been found to shrink the volume of the aforementioned antennas by $40 \%$ and $60 \%$, respectively [1]. Other MICS implantable anter $\equiv$ pplying similar miniaturization techniques include a 3457- $\mathrm{nm}^{3}$ PIFA with a serpentin $\equiv$ iator built on MACOR substrate $\left(\varepsilon_{r}=6.1\right)$ [8], and a $6480-\frac{\bar{\pi}^{3}}{3}$ antenna with a waffletype radiator built on silicon substrate $\left(\varepsilon_{r}=3.1\right)$ [9], both intended for generic body implantation (2/3 human muscle properties used to represent average body properties). Multilayer structures including vertically stacked radiating patches increase the length of the current flow and further assist in miniaturization. Reported skin-implantable stacked PIFAs occupy miniaturized volumes of 598 [10], 383 [11], 337 [12], and $32.7 \mathrm{~mm}^{3}$ [5], [13]. Biocompatibility issues entail the use of a superstrate for all structures, while gain degradation with size reduction must also be considered.

Numerical models of implantable patch antennas proposed in the literature are generally simplified; zero-thickness perfectly conducting sheet model for the radiating and ground planes, glue used to bond the lay $\equiv$ ogether is not taken into account, while ideal models of $50=\overline{\bar{\nu}}$ oaxial cables are used to feed the structures. Most studies report reflection coefficient measurements (e.g., [1], [2], [8]-[11]) without assessing the effect of fabrication/testing details on the resonance of the simplified antenna or discussing design refinements required to restore the desired performance. Only influence of the feeding network has been discussed for patch implantable antennas [14]; however, no design modifications were suggested to overcome its effects in antenna resonance. Preliminary investigations on metallization, gluing, and feeding considerations in numerical design have recently been reported for 3-D cylindrical antennas [15]. As the antenna dimensions shrink, the effect of fabrication issues becomes even more critical. 
In this paper, the first challenge lies in proposing a novel design-and-testing methodology that optimizes the design of simplified implantable antennas to suit-specific prototype fabrication approaches. Antenna design is optimized based on quasiNewton optimization [16] to address metallization, gluing, and feeding considerations, while sensitivity tests are performed to determine the maximum allowable deviations between numerical and experimental results. A low-cost technique is further suggested for reliably measuring the electric properties of (tissuesimulating) liquids without the use of commercial equipment.

The second challenge lies in dealing with prototype fabrication of miniature antennas for which tolerance to fabrication issues (e.g., soldering bumps, uncertainties in glue thickness and permittivity, etc.) is considered to be highly critical. A parametric model of a novel miniature antenna is proposed for skin implantation, and the design-and-testing methodology is applied to optimally adjust it within a specific fabrication/testing approach, for validation purposes. To speed up design while providing an antenna model for generic skin implantation (e.g., inside the head, arm, and trunk for intracranial pressure, blood pressure, and glucose monitoring, respectively [17]), investigations are performed inside a canonical skin-tissue model. Antenna performance is finally evaluated inside an anatomical head model (e.g., pressure monitoring, brain wave sensing, stroke rehabilitation, etc.).

An attempt is, thus, made to gain valuable insight into prototype fabrication and testing considerations that need to be considered within simulations for miniature implantable patch antennas. Although skin implantation in the MICS band is emphasized, the proposed methodology can easily be applied to optimize antennas for several other implantation scenarios and operation frequencies [18], [19]. This paper is organized as follows. Section II describes the proposed methods and models. Validation is performed in Section III. Numerical results inside an anatomical head model are presented in Section IV. This paper concludes in Section V.

\section{MODELS AND METHODS}

\section{A. Tissue Models and Numerical Methods}

To speed up simulations while providing a generic skinimplantable antenna, design, a canonical skin-tissue model [see Fig. 1(a)] [5], [17]. The dimensions are those of a typical semifilled plastic drinking glass, while the antenna is considered to be immersed by $2 \mathrm{~cm}$. Antenna resonance has been found to be almost insensitive to the shape of the tissue model, as long as it is surrounded by skintissue. Finite element (FE) simulations are carried out using the commercial software Ansoft HFSS [20]. The FE solver performs iterative tetrahedron-meshing refinement automatically with the mesh being perturbed by $30 \%$ between each pass. The mesh refinement procedure stops when the maximum change in the reflection coefficient magnitude $\left(\left|S_{11}\right|\right)$ between two consecutive passes is less than 0.02 or when the number of passes exceeds 10 .

Performance of the antenna is further examined inside a 13tissue (see Table I) anatomical head model [see Fig. 1(b)] [5].

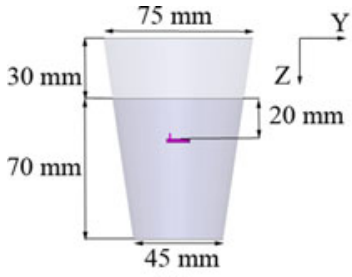

(a)

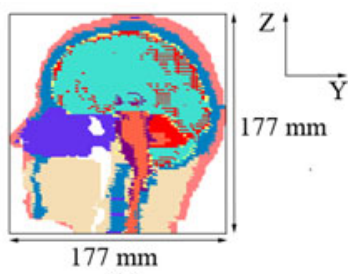

(b)
Fig. 1. Tissue models: (a) typical semi-filled plastic drinking glass and (b) 13-tissue anatomical human head.

TABLE I

Tissue Electric Properties AT 402 MHz And Mass Densities

\begin{tabular}{c|ccc}
\hline \hline Biological Tissue & $\begin{array}{c}\text { Permittivity } \\
\left(\varepsilon_{\mathrm{r}}\right)\end{array}$ & $\begin{array}{c}\text { Conductivity } \\
(\sigma[\mathrm{S} / \mathrm{m}])\end{array}$ & $\begin{array}{c}\text { Mass Density } \\
\left(\rho\left[\mathrm{kg} / \mathrm{m}^{3}\right]\right)\end{array}$ \\
\hline skin (dry) & 46.74 & 0.69 & 1100 \\
bone (cortical) & 13.10 & 0.09 & 2200 \\
dura & 46.65 & 0.83 & 1100 \\
cerebrospinal fluid & 70.97 & 2.25 & 1020 \\
grey matter & 57.40 & 0.74 & 1030 \\
white matter & 42.05 & 0.45 & 1030 \\
muscle & 57.11 & 0.80 & 1040 \\
cartilage & 45.45 & 0.59 & 1100 \\
vitreous humor & 69.00 & 1.53 & 1000 \\
lens & 48.14 & 0.67 & 1100 \\
eye sclera & 57.66 & 1.00 & 1100 \\
spinal cord & 35.39 & 0.45 & 1040 \\
cerebellum & 55.94 & 1.03 & 1030 \\
\hline \hline
\end{tabular}

The antenna is implanted $3.6 \mathrm{~mm}$ under the skin, with its ground 138 plane being placed in parallel with the horizontal plane of the 139 head model. Finite-difference time-domain (FDTD) simulations 140 are carried out in Remcom XFDTD [21], which enables efficient 141 modeling of anatomical body parts. The antenna and anatomi- 142 cal head a $\equiv$ deled in $0.1-$ and 3.66- $\mathrm{mm}^{3}$ cells. Cells of $5 \mathrm{~mm} 143$ in edge $\Delta \bar{X}$ model free space $\equiv$ to meet the FDTD spatial 144 step constraint $\left(\Delta x<\lambda_{\min } / 10\right) \overline{2}$ mere $\lambda_{\text {min }}$ indicates the wave- 145 length of the highest frequency of interest) for the simulation 146 set up under consideration. This sets the maximum simulation 147 frequer $\equiv$ ax to $6 \mathrm{GHz}\left(f_{\max }<c /(10 \Delta x)\right.$, where $c$ is the speed 148 of light $\sqrt{\mathrm{a} a} d$ the time step $\Delta t$ to $9.622 \mathrm{ps}(\Delta t=\Delta x / c \sqrt{3})$, as 149 referenced to free space. Meshing is adaptive to avoid abrupt 150 transitions. A sinusoidal and a Gaussian source (pulse width of 151 32 time steps) are used in the single-frequen $\equiv$ d broadband 152 simulations. Calculations continue up to a $30-\overline{\mathrm{BB}}$ convergence. 153

Absorbing boundaries are set $\lambda_{0} / 4$ ( $\lambda_{0}$ is the free-space wave- 154 length, $f_{0}=402 \mathrm{MHz}$ ) away from all simulation setups in order 155 to extend radiation infinitely far, while guaranteeing stability of 156 the numerical calculations [20], [21]. Tissue electric properties 157 at $402 \mathrm{MHz}$ are considered (se $\equiv$ le I [22]), and approximated 158 as constant inside the 300-500-K $\mathrm{Hz}$ frequency range [5]. Tissue 159 mass densities are also provided in Table I.

\section{B. Parametric Antenna Model}

A parametric model of a miniature stacked PIFA is proposed 162 for skin implantation, as shown in Fig. 2.

Corresponding dimensions are indicated in Table II for a simplified antenna (simplified), an optimized antenna considering 159
160 (n)

\section{.}

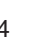

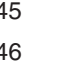
7 (n)

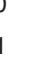

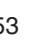

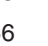

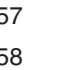




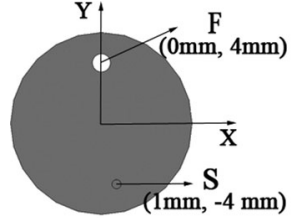

(a)

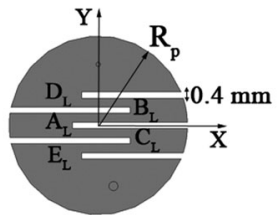

(b)

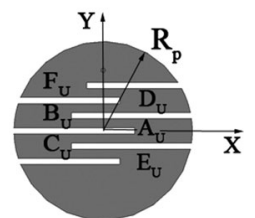

(c)

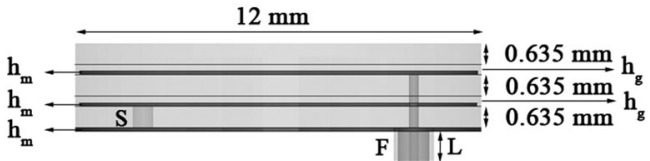

(d)

Fig. 2. Proposed parametric antenna model: (a) ground plane, (b) lower patch, (c) upper patch, and (d) side view.

TABLE II

VARIABLE VALUES OF THE PROPOSED SIMPLIFIEU AND FABRICATION-SPECIFIC ANTENNAS (IN mm)

\begin{tabular}{c|c|c|c}
\hline \hline & simplified & fabrication-specific & head-tuned \\
\hline $\mathrm{h}_{\mathrm{m}}$ & 0 & 0.017 & 0 \\
$\varepsilon_{\mathrm{rg}}$ & - & 2 & - \\
$\mathrm{h}_{\mathrm{g}}$ & 0 & 0.3 & 0 \\
coax type & simplified & $\mathrm{EZ}-47$ & simplified \\
$\mathrm{L}$ & 1 & 50 & 1 \\
$\mathrm{R}_{\mathrm{p}}$ & 5 & 5.9 & 5 \\
$\mathrm{x}_{\mathrm{Al}}$ & -1.3 & -1.7 & -2.0 \\
$\mathrm{X}_{\mathrm{Bl}}$ & 3.8 & 4.3 & 3.8 \\
$\mathrm{x}_{\mathrm{Cl}}$ & 3.8 & 4.3 & 3.8 \\
$\mathrm{x}_{\mathrm{Dl}}$ & 2.5 & 2.6 & 2.5 \\
$\mathrm{x}_{\mathrm{El}}$ & 2.5 & 2.6 & 2.5 \\
$\mathrm{x}_{\mathrm{Au}}$ & 4.1 & 5.5 & 4.1 \\
$\mathrm{x}_{\mathrm{Bu}}$ & -3.8 & -5.3 & -3.8 \\
$\mathrm{x}_{\mathrm{Cu}}$ & -3.8 & -5.3 & -3.8 \\
$\mathrm{X}_{\mathrm{Du}}$ & 2.5 & 4.9 & 3.4 \\
$\mathrm{x}_{\mathrm{Eu}}$ & 2.5 & 4.9 & 3.4 \\
$\mathrm{x}_{\mathrm{Fu}}$ & -2.4 & -3.8 & -2.4 \\
\hline \hline
\end{tabular}

specific fabrication issues (fabrication थpecific), and a fine-tuned version of the simplified antenna inside the anatomical head model of Fig. 1(b) (head-tuned). Circular shape is chosen to avoid sharp edges, while the origin of the coordinate system is considered to be located at the $\Rightarrow$ of the PIFA ground plane. The model consists of a 6-mm $\overline{\bar{r}}$ anus ground plane and two $R_{p}$-radius vertically stacked, meandered patches. Copper sheets with a thickness of $h_{m}$ are considered for the ground, $\equiv$ r, and upper patches. Both patches are fed by an $L$-length, $50-82$ coaxial cable ( $\mathrm{F}: x=0 \mathrm{~mm}, y=4 \mathrm{~mm}$ ), and radiate. Each one is printed on a $0.635-\mathrm{mm}$ substrate (lower/upper), while a $0.635-\mathrm{mm} \mathrm{su}$ perstrate covers the structure to preserve its biocompatibility and robustness. Rogers RO $3210\left(\varepsilon_{r}=10.2, \tan \delta=0.003\right)$, which has long been used in implantable antennas [1], [2], [10]-[12] is chosen as the dielectric material. Glue layers $\left(\varepsilon_{r}=\varepsilon_{r g}\right)$ with a thickness of $h_{g}$ bond the dielectric layers together. Meanders are equidistant by $1 \mathrm{~mm}$, and their lengths are denoted by the $x$ coordinate $x_{i j}$, where the subscripts $\{i j: i=A-F$, and $j=$ $L, U\}$ identify the meander in Fig. 2(b) and (c). Their width is designed to be small $(0.4 \mathrm{mr} \equiv$ as to maximize the area of the patch for radiation. A 0.3 -mmrradius shorting pin $(S: x=1 \mathrm{~mm}$,
TABLE III

EFFect of Metallization, GLuing, AND FEEding on the ResonanCE OF THE PROPOSED SIMPLIFIED ANTENNA

\begin{tabular}{ccccc|cc}
\hline \hline $\mathrm{h}_{\mathrm{m}}[\mathrm{mm}]$ & $\varepsilon_{\mathrm{rg}}$ & $\mathrm{h}_{\mathrm{g}}[\mathrm{mm}]$ & coax type & $\mathrm{L}[\mathrm{mm}]$ & $\mathrm{f}_{\mathrm{res}}[\mathrm{MHz}]$ & $\mid \mathrm{S}_{11 @ \text { fres }}[\mathrm{dB}]$ \\
\hline 0 & - & 0 & simplified & 1 & 402 & -46.9 \\
\hline $\mathbf{1 7}$ & - & 0 & simplified & 1 & 403.5 & -24.7 \\
$\mathbf{3 5}$ & - & 0 & simplified & 1 & 406.0 & -23.4 \\
$\mathbf{7 0}$ & - & 0 & simplified & 1 & 409.0 & -19.7 \\
\hline 0 & $\mathbf{3}$ & $\mathbf{0 . 0 5}$ & simplified & 1 & 439.2 & -17.3 \\
0 & $\mathbf{3}$ & $\mathbf{0 . 1}$ & simplified & 1 & 460.3 & -10.6 \\
0 & $\mathbf{3}$ & $\mathbf{0 . 2}$ & simplified & 1 & 507.3 & -6.9 \\
\hline 0 & $\mathbf{2}$ & $\mathbf{0 . 0 5}$ & simplified & 1 & 446.2 & -11.8 \\
0 & $\mathbf{2}$ & $\mathbf{0 . 1}$ & simplified & 1 & 486.2 & -7.8 \\
0 & $\mathbf{2}$ & $\mathbf{0 . 2}$ & simplified & 1 & 546.4 & -4.7 \\
\hline 0 & - & 0 & EZ-47 & 1 & 405.3 & -39.8 \\
\hline 0 & - & 0 & EZ-47 & $\mathbf{1 0}$ & 401.2 & -36.9 \\
0 & - & 0 & EZ-47 & $\mathbf{3 0}$ & 398.0 & -28.4 \\
0 & - & 0 & EZ-47 & $\mathbf{6 0}$ & 398.0 & -32.4 \\
\hline \hline
\end{tabular}

$y=-4 \mathrm{~mm}$ ) connects the ground plane with the lower patch for further miniaturization.

The simplified version of the parametric antenna model exhibits zero thickness, perfect electric ground- and patch-planes, igno $\equiv$ e presence of glue, and is fed by a simplified $L=1 \mathrm{~mm}$, $50-\Omega \overline{k \sigma} \times x i a l$ cable (inner and outer conductors modeled as a perfect conductor cylinder and a zero-thickness perfect conducting sheet, respectively). Variable values shown in Table II under "simplified" are found to achieve a reflection coefficient magnitude $\left(\left|S_{11}\right|\right)$ of be $\equiv$ an $-25 \mathrm{~dB}$ in the MICS band. The effects of metallization $h, \overline{\bar{r}}$, gluing $\left(\varepsilon_{r g}, h_{g}\right.$, ) and feeding (coax type, $\left.L\right)$ are shown in Table III. Resonance characteristics are recorded, i.e., the exhibited resonance frequency $f_{\text {res }}$ and $\left|S_{11 @ \text { fres }}\right|$. Gluing is found to be the most critical factor; low-permittivity glue layers isolate the high-permittivity substrate layers, thus decreasing the effective dielectric constant and electrical length of the antenna, while increasing its resonance frequency.

$\equiv$ realistic prototype fabrication scenario, the metallization $h_{r}$, gluing $\left(\varepsilon_{r g}, h_{g}\right)$, and feeding (coax type, $L$ ) variables are set by the fabrication approach under consideration. Tuning the $R_{p}$ and $x_{i j}$ variables alters the effective dimensions of the antenna and helps achieve the desired resonance characteristics [23].

\section{Design-and-Testing Methodology}

An iterative design-and-testing methodology is proposed for 210 implantable antennas, as summarized in Fig. 3. The basic idea is to optimize numerical antenna design for a specific prototype fabrication procedure and in vitro testing setup.

The simplified version of the parametric antenna model is initially optimized to address fabrication limitations and obtain the fabrication-specific antenna. Metallization $\left(h_{m}\right)$, gluing $\left(\varepsilon_{r g}\right.$, $h_{g}$ ), and feeding (coax type, $L$ ) variables are set to the values specified by the fabrication approach under consideration. The rest of the variables $\left(R_{p}, x_{i j}\right)$ are considered as dimensions in the solution space and are optimized based on quasi-Newton optimization [16]. These are initialized to the $\equiv$ es of the simplified antenna and vary within the range $[5 \mathrm{~mm}, 5.9 \mathrm{~mm}]$ $\left(R_{p}\right)$ and $\left[-\left(R_{p}-0.3 \mathrm{~mm}\right),\left(R_{p}-0.3 \mathrm{~mm}\right)\right]\left(x_{i j}\right)$. The minimum and maximum step values are set to 0.1 and $0.4 \mathrm{~mm}$, respectively. The cost function is defined as the magnitude of the reflection 


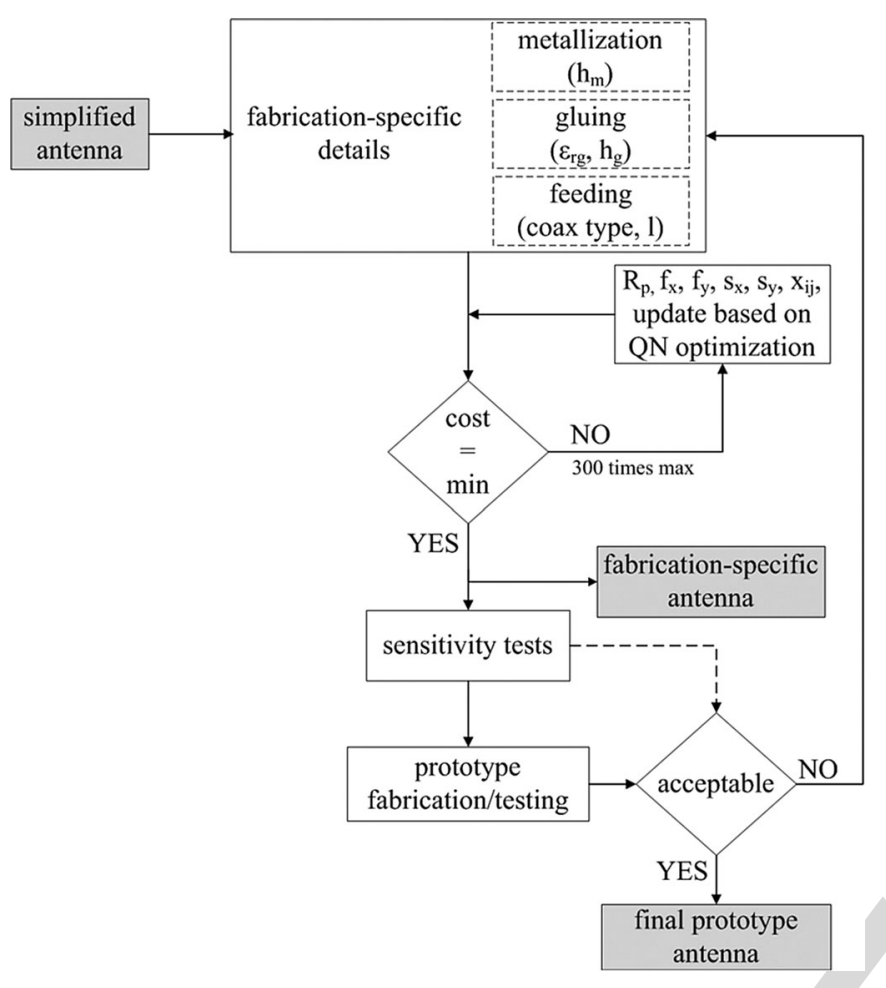

Fig. 3. Proposed design-and-testing methodology for implantable antennas.

coefficient at the desired resonance frequency of $402 \mathrm{MHz}$

$$
\operatorname{cost}=\left|S_{11 @ 402 \mathrm{MHz}}\right| \text {. }
$$

Since the goal is to improve antenna resonance around $402 \mathrm{MHz}$, without aimlessly delaying design, the optimization process terminates when (1) is minimized, or when the number of iterations exceeds 300 .

Numerica $\equiv$ itivity tests are subsequently performed in or-

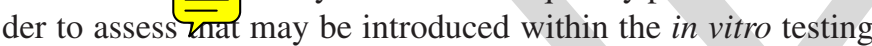
of the fabrication-specific antenna. The effect of minor modifications in the most sensitive antenna design and testing parameters is examined, as imposed by the fabrication approach and measurement setup under consideration. Once the prototype antenna is fabricated and tested, sensitivity tests determine the maximum allowable deviation between numerical and experimental results, and the potential need for further refinement in numerical antenna design. Deviations within the acceptable limits mean that the final prototype antenna has been obtained.

\section{Measurement of the Electric Properties of Liquids}

In vitro testing of implantable antennas inside tissuesimulating liquids requires experimental measurement of the exhibited electric properties $\left(\varepsilon_{r}, \sigma\right)$ to ensure conformance with the numerical values [22]. There exist commercial complex permittivity measurement systems (e.g., Agilent Technologies 85071E); however, alternative approaches are solicited for laboratories that are not equipped with such systems. A novel low-cost and reliable complex permittivity measurement technique is hereafter described and evaluated. To enhance confidence in measurements, the technique is both reflection- and

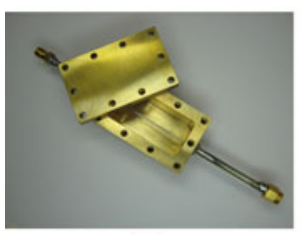

(a)

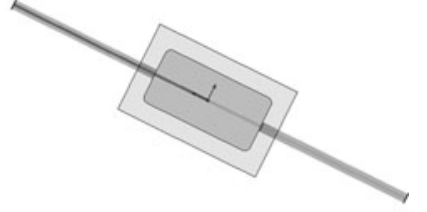

(b)
Fig. 4. (a) Coaxial container for complex permittivity measurement of liquid materials and (b) numerical model.

transmission-based rather than solely reflection-based, as is the usually reported case (e.g., [24] and [25]).

The measurement setup consists of a parallelepiped container intercepted by the inner conductor of a coaxial cable, as shown in Fig. 4(a). The arrangement corresponds to a coaxial container that can be filled with any liquid dielectric material. Its dimensions (exterior container of $52 \mathrm{~mm} \times 32 \mathrm{~mm} \times 32.2 \mathrm{~mm}$, interior cavity of $40 \mathrm{~mm} \times 20 \mathrm{~mm} \times 20 \mathrm{~mm}$ ) have been chosen so as to place a resonance mode around the desired measuring bandwidth of 300-500 MHz when filled with a high-permittivity liquid. After closing the container lid, the structure represents a transition between coaxial guides with a step characteristic impedance discontinuity. The transfer function between the two coaxial connectors outside the container depends upon the complex permittivity of the container's filling liquid. This can be de-embedded by comparing the measured scattering-matrix $(S$ matrix) with FE simulation results for the same structure. The simulation model is shown in Fig. 4(b), and is fine-tuned through experimental measurements for the empty (closed) container. In the case of nonhomogeneous mixtures, the present approach provides inherently an average permittivity value. The liquid is assumed to fill the inner volume of the container completely.

To validate the proposed experimental technique, measure- 275 ments are carried out considering the container to be filled with 276 a liquid with well-known properties, i.e., distilled water. In the 277 simulations, the complex relative permittivity of distilled water 278 is approximated by the Debye model as a function of frequency

$$
\varepsilon=\varepsilon_{r}^{\prime}-j \varepsilon_{r}^{\prime \prime}=\varepsilon_{\infty}+\frac{\varepsilon_{s}-\varepsilon_{\infty}}{1+j \omega \tau}
$$

where $\varepsilon_{\infty}=4.6$ is the optical permittivity at high frequencies, 280 $\varepsilon_{s}=78.3$ is the static permittivity at low frequencies, and $\tau 281$ $=8.07 \mathrm{ps}$ is the electrical relaxation time [25]. Numerical and 282 experimental results are superimposed in Fig. 5 indicating quite 283 good agreement.

\section{VALIDATION}

Validation of the proposed design-and-testing methodology 286 is performed within the framework of a specific fabrication process, as dictated by the available materials, assembling tools, and technical expertise/experience.

\section{A. Prototype Fabrication Approach}

Standard 0.017- $\overline{\mathrm{mm}} \equiv$ electrodeposited copper foil covers 291 both sides of Rogers RV 3210. The sheets are etched using a 292 photolithographic process. The lower substrate layer contains 293 208 29

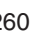
(1)

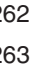
tos (a)

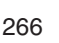

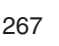

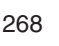
269 270 271 272 273 .

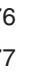

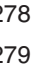




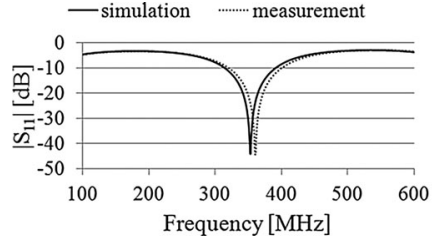

(a)

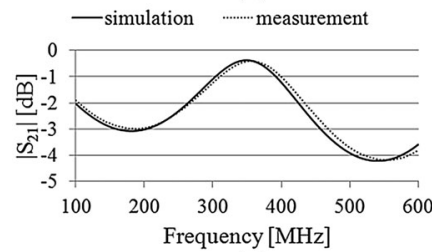

(c)

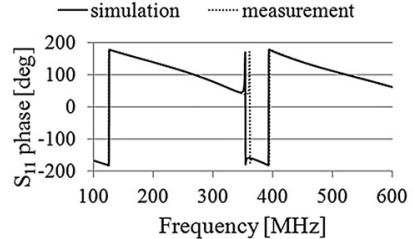

(b)

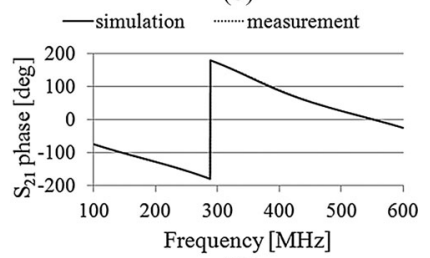

(d)

Fig. 5. Results for the coaxial container filled with distilled water: (a) magnitude of $S_{11}\left(\left|S_{11}\right|\right)$, (b) phase of $S_{11}$ (wrapped), (c) magnitude of $S_{21}\left(\left|S_{21}\right|\right)$, and (d) phase of $S_{21}$ (wrapped).

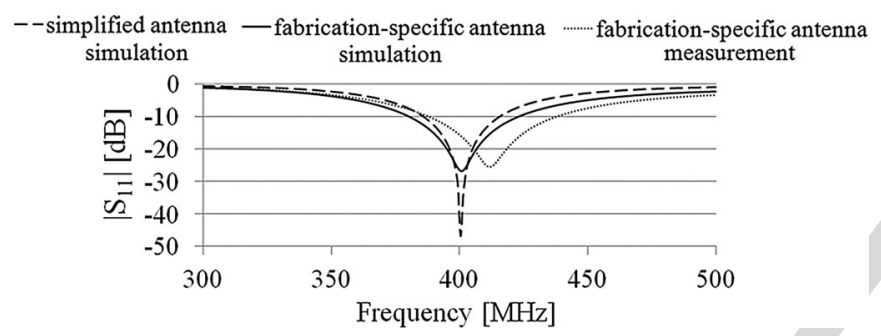

Fig. 6. Simulated and measured reflection coefficient frequency response of the simplified and fabrication-specific antennas.

the ground plane and the lower patch, the upper substrate contains the upper patch, and the superstrate has no metallization. Sprayable glue $3 \mathrm{M} 77$ is used to bond the three layers $\left(\varepsilon_{r}=2\right)$, which is found to exhibit an average thickness of $0.3 \mathrm{~mm}$ for th $\equiv$ cific fabrication $\equiv$ ess. The antenna is fed through a 50-Йmlong EZ-47 semingld coaxial cable.

\section{B. Validation}

1) Fabrication-Specific Antenna: Metallization $\left(h_{m}\right)$, gluing $\left(\varepsilon_{r g}, h_{g}\right)$, and feeding (coaxial type, $\left.L\right)$ variables are set to the values imposed by the available fabrication approach, while the $R_{p}$ and $x_{i j}$ variables are optimized accordingly. Parameter values shown in Table II under "fabrication-specific" are found to tune the fabrication-specific antenna at $402 \mathrm{MHz}$ with a wide 10-dB bandwidth (defined at $\left|S_{11}\right|<-10 \mathrm{~dB}$ ) of $44 \mathrm{MHz}$ covering the MICS band. Radii of the patches and meander lengths are significantly increased as compared to the simplified antenna. The aim is to counteract the increase in resonance frequency imposed by the low-permittivity glue layers. The simulated reflection coefficient frequency responses of the simplified and fabrication-specific antennas are shown in Fig. 6.

2) Sensitivity Tests: Sensitivity test results related to antenna design and experimental phantom uncertainties are indicated in Figs. 7 and 8, respectively. Only the antenna or phantom parameter under investigation is considered variable in each case, while all other parameters are kept constant and equal to those of the fabrication-specific antenna design (see Table II, fabricationspecific) and of the theoretical tissue model [see Fig. 1(a)].

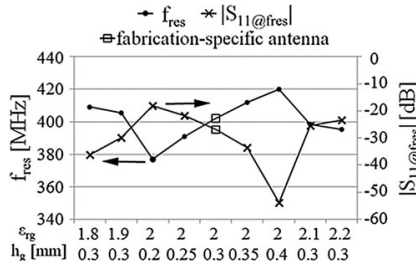

(a)

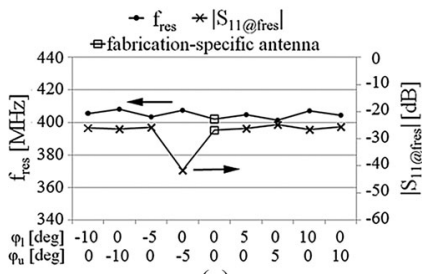

(c)

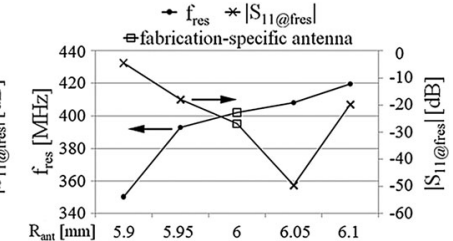

(b)

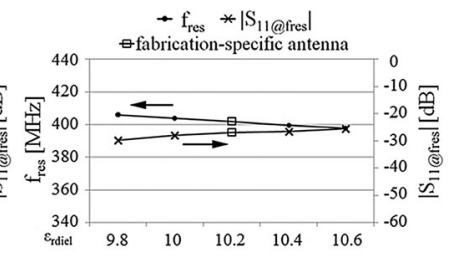

(d)
Fig. 7. Sensitivity test results $\left(f_{\text {res }},\left|S_{11 @ \text { fres }}\right|\right)$ rel $\bar{\equiv}$ prototype antenna parame $\bar{\equiv}$ a) glui $\bar{\equiv}, h_{g}$ ), (b) antenna radius $\overline{F_{\text {ant }}}$, (c) rotation of the lower $\varphi$ iand $\bar{\equiv}$ paches, and (d) permittivity of the Rogers 3210 dielectric material, $\varepsilon_{r}$

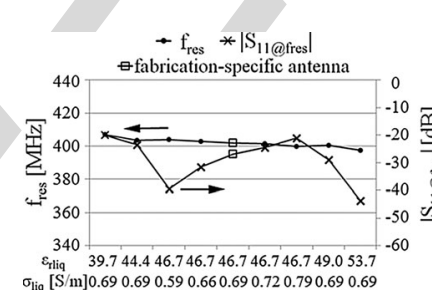

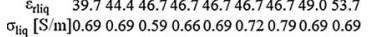

(a)

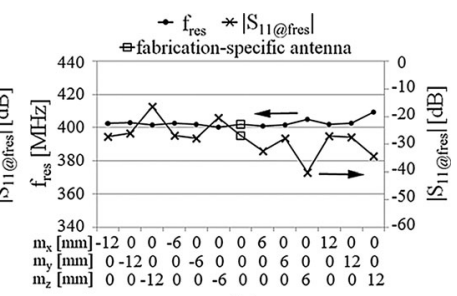

(b)
Fig. 8. Sensitivity test results $\left(f_{\text {res }},\left|S_{11 @ \text { fres }}\right|\right)$ related to phantom parameters: (a) permittivity $\varepsilon_{r \text { liq }}$ and conductivity $\sigma_{\text {liq }}$ of the skin-emulating liquid and (b) relative shift of the phantom from its original position $\left(m_{x}, m_{y}, m_{z}\right)$.

Resonance characteristics including the resonance frequency $f_{\text {res }}$ and $\left|S_{11 @ \text { fres }}\right|$ are recorded, while the performance of the fabrication-specific antenna is also shown for reference. Given the fabrication approach described in Section III-A, the following parameters are identified as potential sources of experimental uncertainties, and examined.

1) Gluing $\left(\varepsilon_{r g}, h_{g}\right)$ [see Fig. $7(a)$ ]. Air bubbles accumulating within the glue prevent $\varepsilon_{r g}$ from being accurately determined. Furthermore, the adopted layer bonding process does not allow fine control of $h_{g}$. This is impaired not only by the glue itself, but also by the slight bump of the microsolder near the coaxial cable and the shorting pin that prevents perfect contact between the layers. Deviations of $\pm 10 \%$ and $33 \%$ in $\varepsilon_{r g}$ and $h_{g}$ are found to cause frequency detunings by up to $1.7 \%$ and $6.2 \%$, respectively;

2) Antenna radius $R_{\text {ant }}[$ see Fig. 7(b)]. Rogers 3210 requires significant mechanical stress (vertical pressure and torsion) for detaching the excess alignment material, degrading accuracy of the cutting procedure. A $0.2=n \mathrm{~m}$ increase $\equiv$ nt detunes the antenna by $4.4 \%$, whereas a 0.1 -mmecrease brings the copper patch sheets in direct contact with the tissue, thus, significantly degrading antenna resonance;

3) Relative rotation between the patches (indicated by the 344 rotation of the lower $\varphi_{l}$ and upper $\varphi_{u}$ patches around 345 
the z-axis) [see Fig. 7(c)]. Even though alignment marks are included in the photolithography masks, the alignment setup is relatively relaxed with respect to angular misalignment of the layers. Misalignment by $10^{\circ}$ is found to cause a maximum frequency detuning of only $1.2 \%$, thus, proving to be of minor importance. This justifies our choice for a relatively flexible alignment approach, while indicating the potential of relaxing the complexity of the assembling setup in order to benefit the gluing process that has been shown to be very critical. Positive and negative rotation angles correspond to clockwise and counterclockwise rotation around the $z$-axis, respectively;

4) Permittivity of the Rogers 3210 dielectric material, $\varepsilon_{\mathrm{rdiel}}$ [see Fig. 7(d)]. The typical value of the Rogers 3210 permittivity is defined to be 10.2 at $10 \mathrm{GHz}$ under $23{ }^{\circ} \mathrm{C}$. Frequency and temperature variations may slightly affect $\varepsilon_{\text {rdiel }}$ and degrade antenna performance. However, sensi tivity tests indicate minor effects in antenna resonance variations of \pm 0.4 in $\varepsilon_{\text {rdiel }}$ may lead to frequency detunings by up to only $1 \%$;

5) Permittivity $\varepsilon_{\text {rliq }}$ and conductivity $\sigma_{\text {liq }}$ of the skinsimulating liquid [see Fig. 8(a)]. Time and room temperature may perturb the properties of the mixture from their nominal values. Changes in $\varepsilon_{r \text { liq }}$ and $\sigma_{\text {liq }}$ by $15 \%$ are found to degrade antenna resonance by up to $1.2 \%$ and $0.5 \%$, respectively;

6) Relative antenna-phantom position (indicated by the relative shift of the phantom $\left(m_{x}, m_{y}, m_{z}\right)$ from its original location) [see Fig. 8(b)]. Since the antenna is manually positioned inside the phantom, slight deviations from the immersion scenario of Fig. 1(b) may occur. However, numerical results indicate insensitivity to antenna positioning inside the phantom as long as it is surrounded by skin-tissue.

3) In Vitro Testing: A skin-tissue-simulating liquid at $402 \mathrm{MHz}$ is prepared (56.18\% sugar, $2.33 \%$ salt, and $41.48 \%$ distilled water [2]) and its electric properties are measured using the technique described in Section II-D. Numerical and measured results are shown in Fig. 9, indicating adequacy of the mixture for in vitro testing. Dispersive, frequency-dependent $\varepsilon_{r}$ and $\sigma$ values of the skin $\equiv$ lating liquid are used in the simulations, as shown in Fig. 10(solid line) [2]. The $\varepsilon_{r}$ and $\sigma$ values of actual skin-tissue are also shown for reference (dotted line) [22].

The prototype antenna is further built [see Fig. 11(a)], connected to a network analyzer, and immersed inside the liquid [see Fig. 11(b)]. The measured reflection coefficient frequency response is shown in Fig. 6 (dotted line). Good agreement exists between numerical and experimental results. A slight resonance shift of $10 \mathrm{MHz}(2.5 \%)$ is observed, which lies within the uncertainty allowances imposed by the sensitivity tests. Nevertheless, both simulation and measurement have an $\left|S_{11}\right|<-10 \mathrm{~dB}$ bandwidth which includes the MICS band.

\section{Performance Inside an Anatomical Head Model}

The proposed antenna model is finally evaluated within the scalp-implantation scenario of Fig. 1(b). The simplified antenna

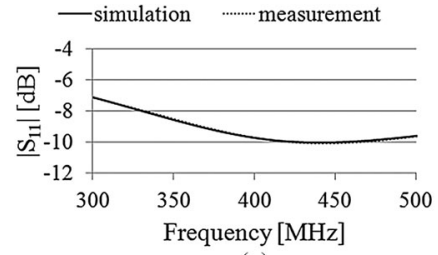

(a)

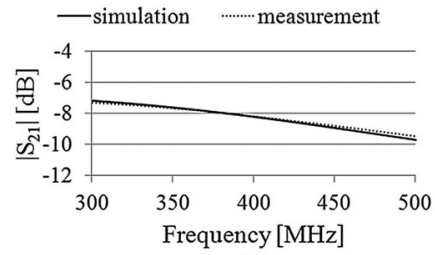

(c)

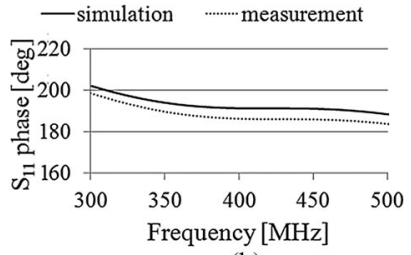

(b)

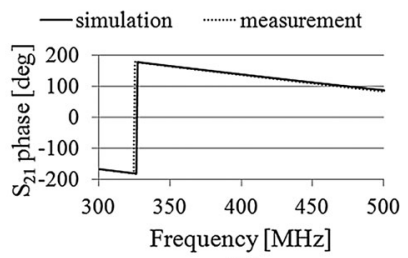

(d)
Fig. 9. Results for the coaxial container filled with skin-emulating liquid: (a) magnitude of $S_{11}\left(\left|S_{11}\right|\right)$, (b) phase of $S_{11}$ (wrapped), (c) magnitude of $S_{21}$ $\left.\left|S_{21}\right|\right)$, and (d) phase of $S_{21}$ (wrapped).

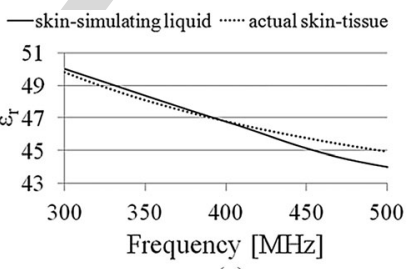

(a)

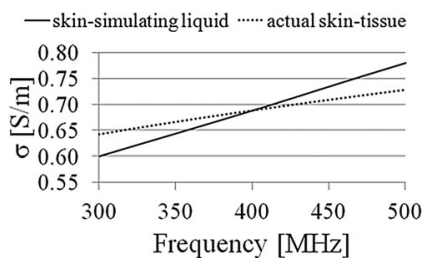

(b)
Fig. 10. Comparison of (a) permittivity $\varepsilon_{r}$ and (b) conductivity $\sigma$ of the skin-emulating liquid with the actual values for skin-tissue.

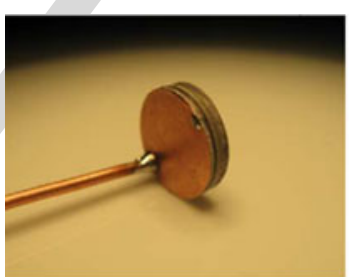

(a)

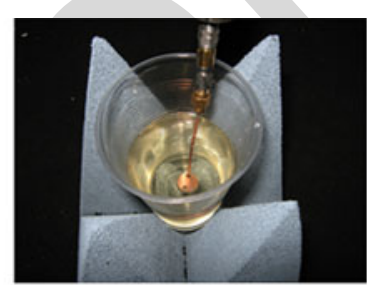

(b)
Fig. 11. (a) Fabricated prototype antenna and (b) in vitro testing setup.

is considered to provide generic results independent of the fab- 401 rication procedure, and fine-tuning is performed (see Table II 402 under "head-tuned") to achieve resonance in the MICS band 403 [see Fig. 12(a)]. Detuning issues for implantable antennas in- 404 side specific anatomical locations have been discussed by the 405 author $\equiv$ 5], [17], and [26]. An inf $\doteq$ imally thin wire and 406

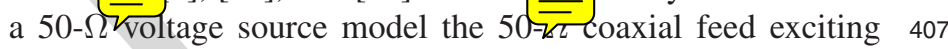
the antenna. The head-tuned antenna radiates an asymmetrical 408 far-field gain radiation pattern [see Fig. 12(b)], with a max- 409 imum gain of $-37.10 \mathrm{dBi}$ exhibited in the $(\theta, \varphi)=\left(110^{\circ}, 410\right.$ $90^{\circ}$ ) direction. Low-gain values are attributed to the small PIFA 411 size $\equiv$ igh-tissue loss. Maximum 1-g-averaged (1-g-avg) and 412 10-g-zaveraged (10-g-avg) specific absorption rate (SAR) val- 413 ues equal 324.74 and $65.09 \mathrm{~W} / \mathrm{kg}$, respectively, for a net in- 414 put power of $1 \mathrm{~W}$. The IEEE C95.1-1999 (1-g-avg SAR $\leq 415$ $1.6 \mathrm{~W} / \mathrm{kg})$ and C95.1-2005 (10-g-avg SAR $\leq 2 \mathrm{~W} / \mathrm{kg})$ safety 416 standards, thus, limit the maximum allowed net input power to 417 4.927 and $30.73 \mathrm{~mW}$, respectively [27]. Local SAR distribution 418 


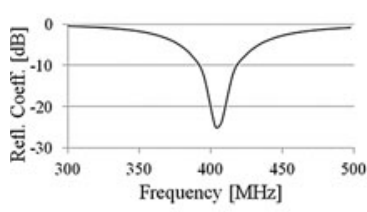

(a)

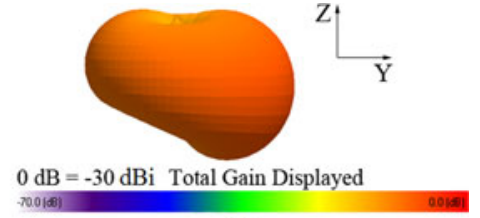

(b)

Fig. 12. (a) Reflection coefficient frequency response and (b) far-field gain radiation pattern of the head-tuned antenna implanted inside the anatomical head model [see Fig. 1(b)].

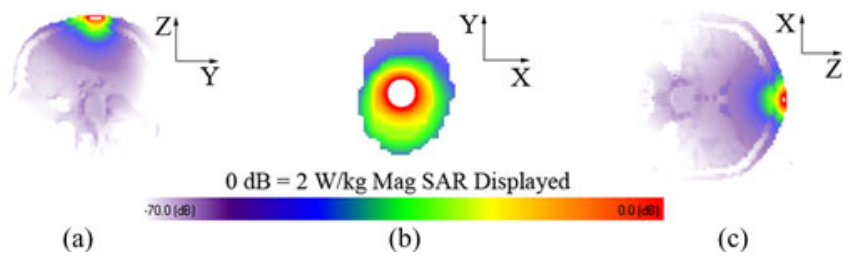

Fig. 13. Local SAR distribution in the (a) $y z$, (b) $x y$, and (c) $z x$ slices of the anatomical head model [see Fig. 1(b)] where maximum local SAR values have been calculated (net input power $=4.927 \mathrm{~mW}$ ).

considering a net-input power of $4.927 \mathrm{~mW}$ is shown in Fig. 13 for the slices where maximum local SAR values have been recorded.

\section{CONCLUSION}

We proposed a parametric model of a skin-implantable miniature PIFA for biotelemetry in the MICS band, and suggested a novel design-and-testing methodology for implantable antennas that incorporates gluing, metallization, and feeding considerations into numerical design. A low-cost, transmission-based technique was also described for reliably measuring the electric properties of liquids, without requiring specific commercial equipment.

Validation was further performed within a specific miniatureantenna-oriented fabrication approach. Sensitivity tests related to antenna design and phantom parameters indicated uncertainties of $0.5-6.2 \%$ in the exhibited resonance frequency, while relative antenna positioning was shown to be of minor significance. A resonance shift of $2.5 \%$ was observed in experimental testing as compared to simulations, which was within the expected uncertainty range.

Antenna implantation inside an anatomical head model required minor design modifications to refine tuning and exhibited an asymmetrical, low-gain (less than $-37.10 \mathrm{~dB}$ ) radiation pattern. Maximum net-input power levels of 4.927 and $30.73 \mathrm{~mW}$ were found to guarantee conformance with the IEEE C95.11999 and C95.1-2005 safety standards.

\section{ACKNOWLEDGMENT}

The authors would like to thank C. Brito and A. Almeida from Instituto Superior Técnico for prototype fabrication and measurement.

\section{REFERENCES}

[1] J. Kim and Y. Rahmat-Samii, "Implanted antennas inside a human body: Simulations, designs and characterizations," IEEE Trans. Microw. Theory Tech., vol. 52, no. 8, pp. 1934-1943, Aug. 2005.

[2] T. Karacolak, A. Z. Hood, and E. Topsakal, "Design of a dual-band implantable antenna and development of skin mimicking gels for continuous glucose monitoring," IEEE Trans. Microw. Theory Tech., vol. 56, no. 4 pp. 1001-1008, Apr. 2008.

[3] R. Warty, M.-R. Tofighi, U. Kawoos, and A. Rosen, "Characterization of implantable antennas for intracranial pressure monitoring: Reflection by and transmission through a scalp phantom," IEEE Trans. Microw. Theory Tech., vol. 56, no. 10, pp. 2366-2376, Oct. 2008.

[4] L. Xu and M. Q.-H. Meng, "Effects of dielectric parameters of human body on radiation characteristics of ingestible wireless device at operating frequency of $430 \mathrm{MHz}$," IEEE Trans. Biomed. Eng., vol. 56, no. 8 pp. 2083-2094, Aug. 2009.

[5] A. Kiourti, M. Christopoulou, and K. S. Nikita, "Performance of a nove miniature antenna implanted in the human head for wireless biotelemetry," in Proc. IEEE Int. Symp. Antennas Propag., Spokane, WA, 2011, pp. 392 395.

[6] "Medical implant communications service (MICS) federal register," Rules Reg., vol. 64, no. 240, pp. 69926-69934, Dec. 1999.

[7] "European Radiocommunications Commission (ERC) Recommendation 70-03 relating to the use of short range devices," presented at the Conf Eur. Postal. Telecomm. Admin. (EPT), 1997, Paper CEPT/ERC 70-03, Annex 12.

[8] P. Soontornpipit, C. M. Furse, and Y. C. Chung, "Design of implantable microstrip antenna for communication with medical implants," IEEE Trans. Microw. Theory Tech., vol. 52, no. 8, pp. 1944-1951, Aug. 2004.

[9] P. Soontornpipit, C. M. Furse, and Y. C. Chung, "Miniaturized biocompatible microstrip antenna using genetic algorithm," IEEE Trans. Antennas Propag., vol. 53, no. 6, pp. 1939-1945, Jun. 2005.

[10] W.-C. Liu, F.-M. Yeh, and M. Ghavami, "Miniaturized implantable broadband antenna for biotelemetry communication," Microw. Opt. Technol. Lett., vol. 50, pp. 2407-2409, Sep. 2008.

[11] W.-C. Liu, S.-H. Chen, and C.-M. Wu, "Bandwidth enhancement and size reduction of an implantable PIFA antenna for biotelemetry devices,' Microw. Opt. Technol. Lett., vol. 51, pp. 755-757, Mar. 2009.

[12] C.-M. Lee, T.-C. Yo, and C.-H. Luo, "Compact broadband stacked implantable antenna for biotelemetry with medical devices," in Proc. IEEE Annu. Wireless Microw. Technol. Conf., Dec. 4-5, 2006, pp. 1-4.

[13] A. Kiourti and K. S. Nikita, "Meandered versus spiral novel $\bar{\equiv}$ ture PIFAs implanted in the human head: Tuning and performance," Tresented at the 2nd ICST Int. Conf. Wireless Mobile Commun. Healthcare, Kos Island, Greece, 2011, to be published.

[14] F. Merli and A. K. Skrivervik, "Design and measurement consideration for implantable antennas for telemetry applications," in Proc. 4th Europ. Conf. Antennas Propag., Barcelona, Spain, Apr. 2010, pp. 1-5.

[15] F. Merli, L. Bolomey, J.-F Zurcher, G. Corradini, E. Meurville, an A. K. Skrivervik, "Design, Realization and measurements of a miniature antenna for implantable wireless communication systems," IEEE Trans. Antennas Propag., vol. 59, no. 10, pp. 3544-3555, Oct. 2011.

[16] W. Sun and Y.-X Yuan, Optimization theory and methods. New York: Springer-Verlag, 2006, ch. 5.

[17] A. Kiourti and K. S. Nikita, "Detuning issues and $\mathrm{p} \equiv$ tance of a novel implantable antenna for telemetry applications," prezcrted at the 6th Europ. Conf. Antennas Propag., Prague, Czech Republic, Mar. 2012.

[18] W. G. Scanlon and J. B. Burns, "Radiowave propagation from a tissue implanted source at $418 \mathrm{MHz}$ and $916.5 \mathrm{MHz}, "$ IEEE Trans. Biomed Eng., vol. 47, no. 4, pp. 527-534, Apr. 2000.

[19] L. C. Chirwa, P. A. Hammond, S. Roy, and D. R. S. Cumming, "Electromagnetic radiation from ingested sources in the human intestine between $150 \mathrm{MHz}$ and $1.2 \mathrm{GHz}, "$ IEEE Trans. Biomed. Eng., vol. 50, no. 4, pp. 484-492, Apr. 2003.

[20] Ansoft High Frequency Structure Simulator (HFSS), Ver. 11, Ansoft Corporation, Pittsburgh, PA, 2008

[21] XFDTD ${ }^{\circledR}$, Electromagnetic Solver Based on the $\bar{\equiv}$ Difference Time Domain Method, Remcom, Inc., State College, PA.

22] C. Gabriel, S. Gabriel, and E. Corthout, "The dielectric properties of biological tissues," Phys. Med. Biol., vol. 41, pp. 2231-2293, 1996.

[23] A. Kiourti, M. Tsakalakis, and K. S. Nikita, "Parar $=$ study and design of implantable PIFAs for wireless biotelemetry," presented at the Proc. 2nd ICST Int. Conf. Wireless Mobile Commun. Healthcare, Kos Island, Greece, 2011. 
[24] D. Popovic, L. McCartney, C. Beasley, M. Lazebnik, M. Okoniewsky, S. C. Hagness, and J. H. Booske, "Precision open-ended coaxial probes for in vivo and ex vivo dielectric spectroscopy of biological tissues at microwave frequencies," IEEE Trans. Microw. Theory Tech., vol. 53, no. 5, pp. 1713-1722, May 2005.

[25] R. Zajicek, L. Oppl, and J. Vrbaf, "Broadband measurement of complex permittivity using reflection method and coaxial probes," Radioengineering, vol. 17, pp. 14-19, Apr. 2008.
[26] A. Kiourti and K. S. Nikita, "Miniature scalp-implantable antennas for telemetry in the MICS and ISM bands: Design, safety $\bar{\equiv}$ rations and 53 . link budget analysis," IEEE Trans. Antennas Propag., tore published.

[27] IEEE Standard for Safety Levels with Respect to Human Exposure to 534 Radiofrequency Electromagnetic Fields, $3 \mathrm{kHz}$ to $300 \mathrm{GHz}$, IEEE Standard 535 C95.1-1999, 2005.

Authors' photographs and biographies not available at the time of publication. 537 
Q1. Author: Citation of "Fig.14" has been changed to "Fig. 13"

Q2. Author: Please provide name of the authors in Refs. [6], [7].

Q3. Author: Please provide the year

Q4. Author: Please update Ref. [26]. 


\title{
Miniature Implantable Antennas for Biomedical Telemetry: From Simulation to Realization
}

\author{
Asimina Kiourti*, Student Member, IEEE, Jorge R. Costa, Senior Member, IEEE, \\ Carlos A. Fernandes, Senior Member, IEEE, André G. Santiago, and Konstantina S. Nikita, Senior Member, IEEE
}

\begin{abstract}
5 Abstract-We address numerical versus experimental design and testing of miniature implantable antennas for biomedical telemetry in the medical implant communications service band $(402-405 \mathrm{MHz})$. A model of a novel miniature antenna is initially proposed for skin implantation, which includes varying parameters to deal with fabrication-specific details. An iterative designand-testing methodology is further suggested to determine the parameter values that minimize deviations between numerical and experimental results. To assist in vitro testing, a low-cost technique is proposed for reliably measuring the electric properties of liquids without requiring commercial equipment. Validation is performed within a specific prototype fabrication/testing approach for miniature antennas. To speed up design while providing an antenna for generic skin implantation, investigations are performed inside a canonical skin-tissue model. Resonance, radiation, and safety performance of the proposed antenna is finally evaluated inside an anatomical head model. This study provides valuable insight into the design of implantable antennas, assessing the significance of fabrication-specific details in numerical simulations and uncertainties in experimental testing for miniature structures. The proposed methodology can be applied to optimize antennas for several fabrication/testing approaches and biotelemetry applications.
\end{abstract}

Index Terms-Biomedical telemetry, dielectric measurements, implantable antenna, in vitro, medical implant communications service (MICS) band.

Manuscript received February 14, 2012; revised April 5, 2012; accepted May 25, 2012. Date of publication; date of current version. This work was supported in part by the Fundação para a Ciência e Tecnologia, Portugal, under Project RFID Local PTDC/EEA-TEL/102390/2008. The work of A.K. was supported by the IEEE Antennas and Propagation Society Doctoral Research Award. Asterisk indicates corresponding author.

${ }^{*}$ A. Kiourti is with the School of Electrical and Computer Engineering and the Institute of Communications and Computer Systems, National Technical University of Athens, Athens 15780, Greece (e-mail: akiourti@biosim.ntua.gr).

J. R. Costa is with the Instituto de Telecomunicações and the Departamento de Ciências e Tecnologias da Informação, Instituto Universitário de Lisboa (ISCTE-IUL), Lisboa 1649-026, Portugal (e-mail: Jorge.Costa@1x.it.pt).

C. A. Fernandes is with Instituto de Telecomunicações and the Instituto Superior Técnico, Technical University of Lisbon, Lisboa 1049-001, Portugal (e-mail: carlos.fernandes@1x.it.pt).

A. G. Santiago is with Instituto Superior Técnico, Technical University of Lisbon, Lisboa 1049-001, Portugal (e-mail: andre.santiago.1988@gmail.com).

K. S. Nikita is with the School of Electrical and Computer Engineering and the Institute of Communications and Computer Systems, National Technical University of Athens, Athens 15780, Greece (e-mail: knikita@ece.ntua.gr).

Color versions of one or more of the figures in this paper are available online at http://ieeexplore.ieee.org.

Digital Object Identifier 10.1109/TBME.2012.2202659 equipment is most commonly performed in the medical implant communications service (MICS) band (402-405 MHz), which is regulated by the United States Federal Communications Commission [6] and the European Radiocommunications Committee [7]. Patch designs are preferred for implant-integrated antennas because of their flexibility in design, conformability, and shape [8].

Design of implantable patch antennas operating in the lowfrequency MICS band draws high-scientific interest to deal with miniaturization. The aim is to decrease the antenna physical size, while increasing its electrical size. Rectangular 10 240- and $5760-\mathrm{mm}^{3}$ chest-implantable antennas have been reported [1], which use the high-permittivity $\left(\varepsilon_{r}=10.2\right)$ Rogers 3210 dielectric and apply a spiral radiator for size reduction. Addition of a shorting pin, thus, conversion to a planar inverted-F antenna (PIFA) acts like a ground plane on a monopole, and has been found to shrink the volume of the aforementioned antennas by $40 \%$ and $60 \%$, respectively [1]. Other MICS implantable antennas applying similar miniaturization techniques include a $3457-\mathrm{mm}^{3}$ PIFA with a serpentine radiator built on MACOR substrate $\left(\varepsilon_{r}=6.1\right)$ [8], and a $6480-\mathrm{mm}^{3}$ antenna with a waffletype radiator built on silicon substrate $\left(\varepsilon_{r}=3.1\right)$ [9], both intended for generic body implantation (2/3 human muscle properties used to represent average body properties). Multilayer structures including vertically stacked radiating patches increase the length of the current flow and further assist in miniaturization. Reported skin-implantable stacked PIFAs occupy miniaturized volumes of 598 [10], 383 [11], 337 [12], and $32.7 \mathrm{~mm}^{3}$ [5], [13]. Biocompatibility issues entail the use of a superstrate for all structures, while gain degradation with size reduction must also be considered.

Numerical models of implantable patch antennas proposed in the literature are generally simplified; zero-thickness perfectly conducting sheet model for the radiating and ground planes, glue used to bond the layers together is not taken into account, while ideal models of 50- $\Omega$ coaxial cables are used to feed the structures. Most studies report reflection coefficient measurements (e.g., [1], [2], [8]-[11]) without assessing the effect of fabrication/testing details on the resonance of the simplified antenna or discussing design refinements required to restore the desired performance. Only influence of the feeding network has been discussed for patch implantable antennas [14]; however, no design modifications were suggested to overcome its effects in antenna resonance. Preliminary investigations on metallization, gluing, and feeding considerations in numerical design have recently been reported for 3-D cylindrical antennas [15]. As the antenna dimensions shrink, the effect of fabrication issues becomes even more critical. 
In this paper, the first challenge lies in proposing a novel design-and-testing methodology that optimizes the design of simplified implantable antennas to suit-specific prototype fabrication approaches. Antenna design is optimized based on quasiNewton optimization [16] to address metallization, gluing, and feeding considerations, while sensitivity tests are performed to determine the maximum allowable deviations between numerical and experimental results. A low-cost technique is further suggested for reliably measuring the electric properties of (tissuesimulating) liquids without the use of commercial equipment.

The second challenge lies in dealing with prototype fabrication of miniature antennas for which tolerance to fabrication issues (e.g., soldering bumps, uncertainties in glue thickness and permittivity, etc.) is considered to be highly critical. A parametric model of a novel miniature antenna is proposed for skin implantation, and the design-and-testing methodology is applied to optimally adjust it within a specific fabrication/testing approach, for validation purposes. To speed up design while providing an antenna model for generic skin implantation (e.g., inside the head, arm, and trunk for intracranial pressure, blood pressure, and glucose monitoring, respectively [17]), investigations are performed inside a canonical skin-tissue model. Antenna performance is finally evaluated inside an anatomical head model (e.g., pressure monitoring, brain wave sensing, stroke rehabilitation, etc.).

An attempt is, thus, made to gain valuable insight into prototype fabrication and testing considerations that need to be considered within simulations for miniature implantable patch antennas. Although skin implantation in the MICS band is emphasized, the proposed methodology can easily be applied to optimize antennas for several other implantation scenarios and operation frequencies [18], [19]. This paper is organized as follows. Section II describes the proposed methods and models. Validation is performed in Section III. Numerical results inside an anatomical head model are presented in Section IV. This paper concludes in Section V.

\section{MODELS AND METHODS}

\section{A. Tissue Models and Numerical Methods}

To speed up simulations while providing a generic skinimplantable antenna, design, and testing are performed inside a canonical skin-tissue model [see Fig. 1(a)] [5], [17]. The dimensions are those of a typical semifilled plastic drinking glass, while the antenna is considered to be immersed by $2 \mathrm{~cm}$. Antenna resonance has been found to be almost insensitive to the shape of the tissue model, as long as it is surrounded by skintissue. Finite element (FE) simulations are carried out using the commercial software Ansoft HFSS [20]. The FE solver performs iterative tetrahedron-meshing refinement automatically with the mesh being perturbed by $30 \%$ between each pass. The mesh refinement procedure stops when the maximum change in the reflection coefficient magnitude $\left(\left|S_{11}\right|\right)$ between two consecutive passes is less than 0.02 or when the number of passes exceeds 10 .

Performance of the antenna is further examined inside a 13tissue (see Table I) anatomical head model [see Fig. 1(b)] [5].

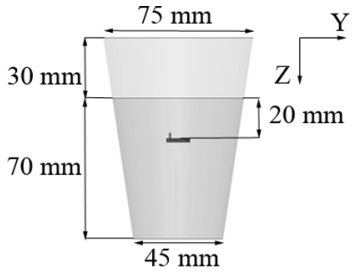

(a)

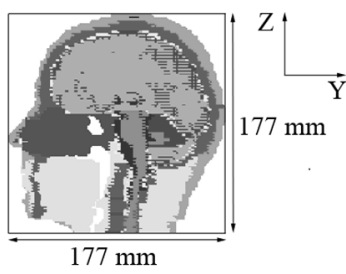

(b)
Fig. 1. Tissue models: (a) typical semi-filled plastic drinking glass and (b) 13-tissue anatomical human head.

TABLE I

Tissue Electric Properties AT 402 MHz And Mass Densities

\begin{tabular}{c|ccc}
\hline \hline Biological Tissue & $\begin{array}{c}\text { Permittivity } \\
\left(\varepsilon_{\mathrm{r}}\right)\end{array}$ & $\begin{array}{c}\text { Conductivity } \\
(\sigma[\mathrm{S} / \mathrm{m}])\end{array}$ & $\begin{array}{c}\text { Mass Density } \\
\left(\rho\left[\mathrm{kg} / \mathrm{m}^{3}\right]\right)\end{array}$ \\
\hline skin (dry) & 46.74 & 0.69 & 1100 \\
bone (cortical) & 13.10 & 0.09 & 2200 \\
dura & 46.65 & 0.83 & 1100 \\
cerebrospinal fluid & 70.97 & 2.25 & 1020 \\
grey matter & 57.40 & 0.74 & 1030 \\
white matter & 42.05 & 0.45 & 1030 \\
muscle & 57.11 & 0.80 & 1040 \\
cartilage & 45.45 & 0.59 & 1100 \\
vitreous humor & 69.00 & 1.53 & 1000 \\
lens & 48.14 & 0.67 & 1100 \\
eye sclera & 57.66 & 1.00 & 1100 \\
spinal cord & 35.39 & 0.45 & 1040 \\
cerebellum & 55.94 & 1.03 & 1030 \\
\hline \hline
\end{tabular}

The antenna is implanted $3.6 \mathrm{~mm}$ under the skin, with its ground 138 plane being placed in parallel with the horizontal plane of the 139 head model. Finite-difference time-domain (FDTD) simulations 140 are carried out in Remcom XFDTD [21], which enables efficient 141 modeling of anatomical body parts. The antenna and anatomi- 142 cal head are modeled in 0.1 - and 3.66- $\mathrm{mm}^{3}$ cells. Cells of $5 \mathrm{~mm} 143$ in edge $\Delta x$ model free space so as to meet the FDTD spatial 144 step constraint $\left(\Delta x<\lambda_{\min } / 10\right)$, where $\lambda_{\min }$ indicates the wave- 145 length of the highest frequency of interest) for the simulation 146 set up under consideration. This sets the maximum simulation 147 frequency $f_{\max }$ to $6 \mathrm{GHz}\left(f_{\max }<c /(10 \Delta x)\right.$, where $c$ is the speed 148 of light, and the time step $\Delta t$ to $9.622 \mathrm{ps}(\Delta t=\Delta x / c \sqrt{3})$, as $\quad 149$ referenced to free space. Meshing is adaptive to avoid abrupt 150 transitions. A sinusoidal and a Gaussian source (pulse width of 151 32 time steps) are used in the single-frequency and broadband 152 simulations. Calculations continue up to a 30-dB convergence. 153

Absorbing boundaries are set $\lambda_{0} / 4\left(\lambda_{0}\right.$ is the free-space wave- 154 length, $f_{0}=402 \mathrm{MHz}$ ) away from all simulation setups in order 155 to extend radiation infinitely far, while guaranteeing stability of 156 the numerical calculations [20], [21]. Tissue electric properties 157 at $402 \mathrm{MHz}$ are considered (see Table I [22]), and approximated 158 as constant inside the 300-500-MHz frequency range [5]. Tissue 159 mass densities are also provided in Table I.

\section{B. Parametric Antenna Model}

A parametric model of a miniature stacked PIFA is proposed 162 for skin implantation, as shown in Fig. 2.

Corresponding dimensions are indicated in Table II for a simplified antenna (simplified), an optimized antenna considering 


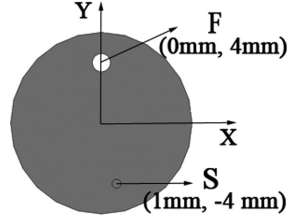

(a)

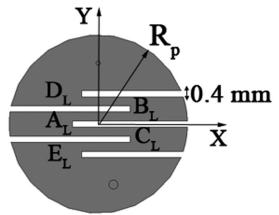

(b)

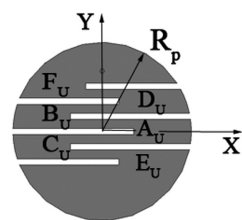

(c)

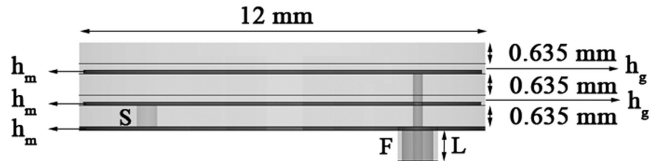

(d)

Fig. 2. Proposed parametric antenna model: (a) ground plane, (b) lower patch, (c) upper patch, and (d) side view.

TABLE II

VARIABLE VALUES OF THE PROPOSED SIMPLIFIED AND FABRICATION-SPECIFIC ANTENNAS (IN mm)

\begin{tabular}{c|c|c|c}
\hline \hline & simplified & fabrication-specific & head-tuned \\
\hline $\mathrm{h}_{\mathrm{m}}$ & 0 & 0.017 & 0 \\
$\varepsilon_{\mathrm{rg}}$ & - & 2 & - \\
$\mathrm{h}_{\mathrm{g}}$ & 0 & 0.3 & 0 \\
coax type & simplified & $\mathrm{EZ}-47$ & simplified \\
$\mathrm{L}$ & 1 & 50 & 1 \\
$\mathrm{R}_{\mathrm{p}}$ & 5 & 5.9 & 5 \\
$\mathrm{x}_{\mathrm{Al}}$ & -1.3 & -1.7 & -2.0 \\
$\mathrm{x}_{\mathrm{Bl}}$ & 3.8 & 4.3 & 3.8 \\
$\mathrm{x}_{\mathrm{Cl}}$ & 3.8 & 4.3 & 3.8 \\
$\mathrm{X}_{\mathrm{Dl}}$ & 2.5 & 2.6 & 2.5 \\
$\mathrm{X}_{\mathrm{El}}$ & 2.5 & 2.6 & 2.5 \\
$\mathrm{x}_{\mathrm{Au}}$ & 4.1 & 5.5 & 4.1 \\
$\mathrm{X}_{\mathrm{Bu}}$ & -3.8 & -5.3 & -3.8 \\
$\mathrm{X}_{\mathrm{Cu}}$ & -3.8 & -5.3 & -3.8 \\
$\mathrm{X}_{\mathrm{Du}}$ & 2.5 & 4.9 & 3.4 \\
$\mathrm{X}_{\mathrm{Eu}}$ & 2.5 & 4.9 & 3.4 \\
$\mathrm{x}_{\mathrm{Fu}}$ & -2.4 & -3.8 & -2.4 \\
\hline \hline
\end{tabular}

specific fabrication issues (fabrication specific), and a fine-tuned version of the simplified antenna inside the anatomical head model of Fig. 1(b) (head-tuned). Circular shape is chosen to avoid sharp edges, while the origin of the coordinate system is considered to be located at the center of the PIFA ground plane. The model consists of a 6-mm radius ground plane and two $R_{p}$-radius vertically stacked, meandered patches. Copper sheets with a thickness of $h_{m}$ are considered for the ground, lower, and upper patches. Both patches are fed by an $L$-length, $50-\Omega$ coaxial cable ( $\mathrm{F}: x=0 \mathrm{~mm}, y=4 \mathrm{~mm}$ ), and radiate. Each one is printed on a $0.635-\mathrm{mm}$ substrate (lower/upper), while a $0.635-\mathrm{mm} \mathrm{su}$ perstrate covers the structure to preserve its biocompatibility and robustness. Rogers RO $3210\left(\varepsilon_{r}=10.2, \tan \delta=0.003\right)$, which has long been used in implantable antennas [1], [2], [10]-[12] is chosen as the dielectric material. Glue layers $\left(\varepsilon_{r}=\varepsilon_{r g}\right)$ with a thickness of $h_{g}$ bond the dielectric layers together. Meanders are equidistant by $1 \mathrm{~mm}$, and their lengths are denoted by the $x$ coordinate $x_{i j}$, where the subscripts $\{i j: i=A-F$, and $j=$ $L, U\}$ identify the meander in Fig. 2(b) and (c). Their width is designed to be small $(0.4 \mathrm{~mm})$ so as to maximize the area of the patch for radiation. A 0.3 -mm radius shorting pin $(S: x=1 \mathrm{~mm}$,
TABLE III

EFFect of Metallization, Gluing, ANd FeEding on the ResonanCE OF THE PROPOSED SIMPLIFIED ANTENNA

\begin{tabular}{ccccc|cc}
\hline \hline $\mathrm{h}_{\mathrm{m}}[\mathrm{mm}]$ & $\varepsilon_{\mathrm{rg}}$ & $\mathrm{h}_{\mathrm{g}}[\mathrm{mm}]$ & coax type & $\mathrm{L}[\mathrm{mm}]$ & $\mathrm{f}_{\mathrm{res}}[\mathrm{MHz}]$ & $\mid \mathrm{S}_{11 @ \text { fres }}[\mathrm{dB}]$ \\
\hline 0 & - & 0 & simplified & 1 & 402 & -46.9 \\
\hline $\mathbf{1 7}$ & - & 0 & simplified & 1 & 403.5 & -24.7 \\
$\mathbf{3 5}$ & - & 0 & simplified & 1 & 406.0 & -23.4 \\
$\mathbf{7 0}$ & - & 0 & simplified & 1 & 409.0 & -19.7 \\
\hline 0 & $\mathbf{3}$ & $\mathbf{0 . 0 5}$ & simplified & 1 & 439.2 & -17.3 \\
0 & $\mathbf{3}$ & $\mathbf{0 . 1}$ & simplified & 1 & 460.3 & -10.6 \\
0 & $\mathbf{3}$ & $\mathbf{0 . 2}$ & simplified & 1 & 507.3 & -6.9 \\
\hline 0 & $\mathbf{2}$ & $\mathbf{0 . 0 5}$ & simplified & 1 & 446.2 & -11.8 \\
0 & $\mathbf{2}$ & $\mathbf{0 . 1}$ & simplified & 1 & 486.2 & -7.8 \\
0 & $\mathbf{2}$ & $\mathbf{0 . 2}$ & simplified & 1 & 546.4 & -4.7 \\
\hline 0 & - & 0 & EZ-47 & 1 & 405.3 & -39.8 \\
\hline 0 & - & 0 & EZ-47 & $\mathbf{1 0}$ & 401.2 & -36.9 \\
0 & - & 0 & EZ-47 & $\mathbf{3 0}$ & 398.0 & -28.4 \\
0 & - & 0 & EZ-47 & $\mathbf{6 0}$ & 398.0 & -32.4 \\
\hline \hline
\end{tabular}

$y=-4 \mathrm{~mm}$ ) connects the ground plane with the lower patch for further miniaturization.

The simplified version of the parametric antenna model exhibits zero thickness, perfect electric ground- and patch-planes, ignores the presence of glue, and is fed by a simplified $L=1 \mathrm{~mm}$, $50-\Omega$ coaxial cable (inner and outer conductors modeled as a perfect conductor cylinder and a zero-thickness perfect conducting sheet, respectively). Variable values shown in Table II under "simplified" are found to achieve a reflection coefficient magnitude $\left(\left|S_{11}\right|\right)$ of better than $-25 \mathrm{~dB}$ in the MICS band. The effects of metallization $h_{m}$, gluing $\left(\varepsilon_{r g}, h_{g}\right.$, ) and feeding (coax type, $L$ ) are shown in Table III. Resonance characteristics are recorded, i.e., the exhibited resonance frequency $f_{\text {res }}$ and $\left|S_{11 @ \text { fres }}\right|$. Gluing is found to be the most critical factor; low-permittivity glue layers isolate the high-permittivity substrate layers, thus decreasing the effective dielectric constant and electrical length of the antenna, while increasing its resonance frequency.

In a realistic prototype fabrication scenario, the metallization $h_{m}$, gluing $\left(\varepsilon_{r g}, h_{g}\right)$, and feeding (coax type, $\left.L\right)$ variables are set by the fabrication approach under consideration. Tuning the $R_{p}$ and $x_{i j}$ variables alters the effective dimensions of the antenna and helps achieve the desired resonance characteristics [23].

\section{Design-and-Testing Methodology}

An iterative design-and-testing methodology is proposed for 210 implantable antennas, as summarized in Fig. 3. The basic idea is to optimize numerical antenna design for a specific prototype fabrication procedure and in vitro testing setup.

The simplified version of the parametric antenna model is initially optimized to address fabrication limitations and obtain the fabrication-specific antenna. Metallization $\left(h_{m}\right)$, gluing $\left(\varepsilon_{r g}\right.$, $h_{g}$ ), and feeding (coax type, $L$ ) variables are set to the values specified by the fabrication approach under consideration. The rest of the variables $\left(R_{p}, x_{i j}\right)$ are considered as dimensions in the solution space and are optimized based on quasi-Newton optimization [16]. These are initialized to the values of the simplified antenna and vary within the range $[5 \mathrm{~mm}, 5.9 \mathrm{~mm}]$ $\left(R_{p}\right)$ and $\left[-\left(R_{p}-0.3 \mathrm{~mm}\right),\left(R_{p}-0.3 \mathrm{~mm}\right)\right]\left(x_{i j}\right)$. The minimum and maximum step values are set to 0.1 and $0.4 \mathrm{~mm}$, respectively. The cost function is defined as the magnitude of the reflection 


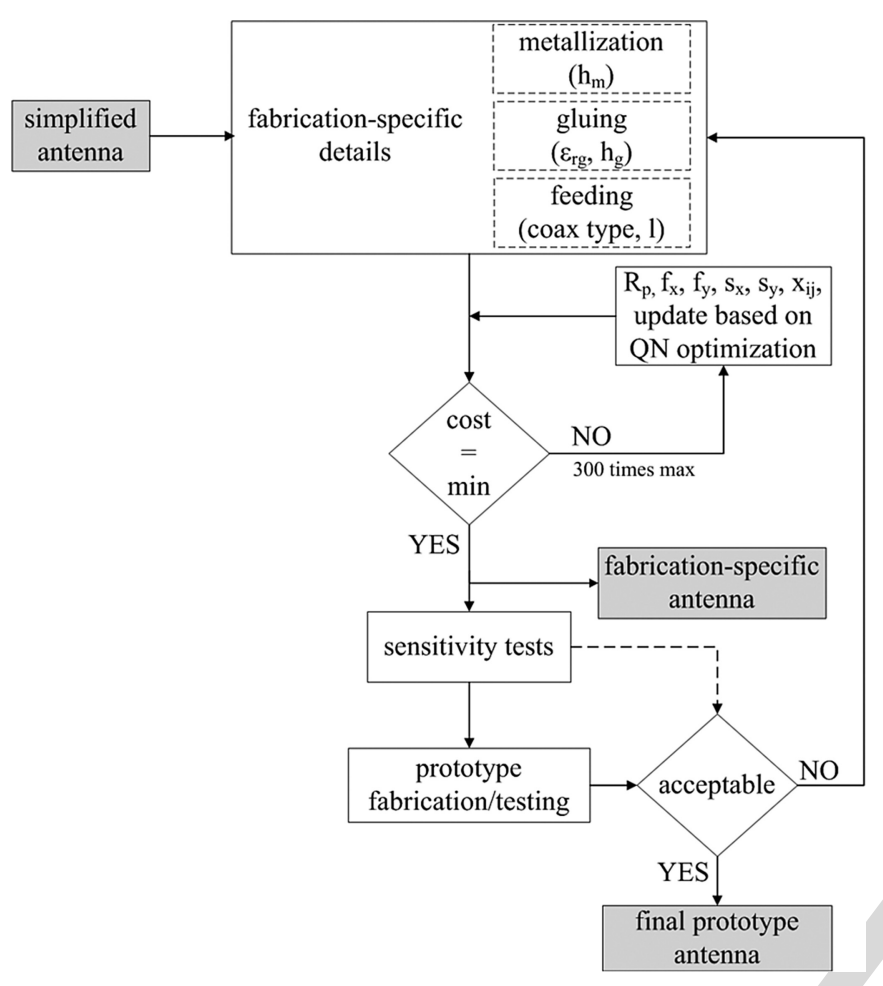

Fig. 3. Proposed design-and-testing methodology for implantable antennas. coefficient at the desired resonance frequency of $402 \mathrm{MHz}$

$$
\operatorname{cost}=\left|S_{11 @ 402 \mathrm{MHz}}\right| .
$$

Since the goal is to improve antenna resonance around $402 \mathrm{MHz}$, without aimlessly delaying design, the optimization process terminates when (1) is minimized, or when the number of iterations exceeds 300 .

Numerical sensitivity tests are subsequently performed in order to assess that may be introduced within the in vitro testing of the fabrication-specific antenna. The effect of minor modifications in the most sensitive antenna design and testing parameters is examined, as imposed by the fabrication approach and measurement setup under consideration. Once the prototype antenna is fabricated and tested, sensitivity tests determine the maximum allowable deviation between numerical and experimental results, and the potential need for further refinement in numerical antenna design. Deviations within the acceptable limits mean that the final prototype antenna has been obtained.

\section{Measurement of the Electric Properties of Liquids}

In vitro testing of implantable antennas inside tissuesimulating liquids requires experimental measurement of the exhibited electric properties $\left(\varepsilon_{r}, \sigma\right)$ to ensure conformance with the numerical values [22]. There exist commercial complex permittivity measurement systems (e.g., Agilent Technologies 85071E); however, alternative approaches are solicited for laboratories that are not equipped with such systems. A novel low-cost and reliable complex permittivity measurement technique is hereafter described and evaluated. To enhance confidence in measurements, the technique is both reflection- and

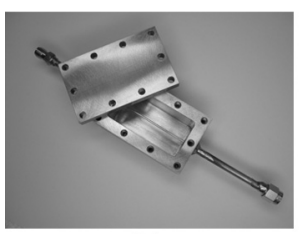

(a)

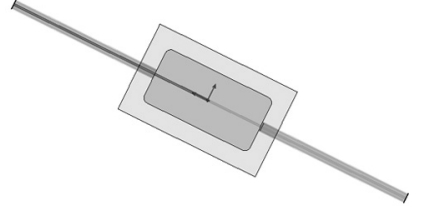

(b)
Fig. 4. (a) Coaxial container for complex permittivity measurement of liquid materials and (b) numerical model.

transmission-based rather than solely reflection-based, as is the usually reported case (e.g., [24] and [25]).

The measurement setup consists of a parallelepiped container intercepted by the inner conductor of a coaxial cable, as shown in Fig. 4(a). The arrangement corresponds to a coaxial container that can be filled with any liquid dielectric material. Its dimensions (exterior container of $52 \mathrm{~mm} \times 32 \mathrm{~mm} \times 32.2 \mathrm{~mm}$, interior cavity of $40 \mathrm{~mm} \times 20 \mathrm{~mm} \times 20 \mathrm{~mm}$ ) have been chosen so as to place a resonance mode around the desired measuring bandwidth of 300-500 MHz when filled with a high-permittivity liquid. After closing the container lid, the structure represents a transition between coaxial guides with a step characteristic impedance discontinuity. The transfer function between the two coaxial connectors outside the container depends upon the complex permittivity of the container's filling liquid. This can be de-embedded by comparing the measured scattering-matrix $(S$ matrix) with FE simulation results for the same structure. The simulation model is shown in Fig. 4(b), and is fine-tuned through experimental measurements for the empty (closed) container. In the case of nonhomogeneous mixtures, the present approach provides inherently an average permittivity value. The liquid is assumed to fill the inner volume of the container completely.

To validate the proposed experimental technique, measure- 275 ments are carried out considering the container to be filled with 276 a liquid with well-known properties, i.e., distilled water. In the 277 simulations, the complex relative permittivity of distilled water 278 is approximated by the Debye model as a function of frequency

$$
\varepsilon=\varepsilon_{r}^{\prime}-j \varepsilon_{r}^{\prime \prime}=\varepsilon_{\infty}+\frac{\varepsilon_{s}-\varepsilon_{\infty}}{1+j \omega \tau}
$$

where $\varepsilon_{\infty}=4.6$ is the optical permittivity at high frequencies, 280 $\varepsilon_{s}=78.3$ is the static permittivity at low frequencies, and $\tau 281$ $=8.07 \mathrm{ps}$ is the electrical relaxation time [25]. Numerical and 282 experimental results are superimposed in Fig. 5 indicating quite 283 good agreement.

\section{VALIDATION}

Validation of the proposed design-and-testing methodology is performed within the framework of a specific fabrication process, as dictated by the available materials, assembling tools, and technical expertise/experience.

\section{A. Prototype Fabrication Approach}

Standard 0.017-mm-thick electrodeposited copper foil covers 291 both sides of Rogers RO 3210. The sheets are etched using a 292 photolithographic process. The lower substrate layer contains 293 271 272 ${ }^{20}$ .

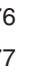
(20) 


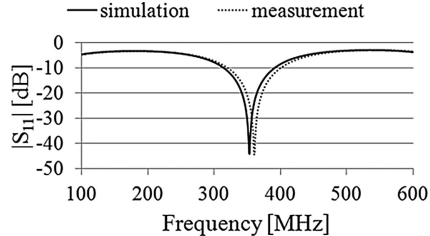

(a)

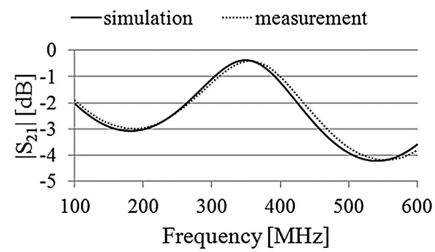

(c)

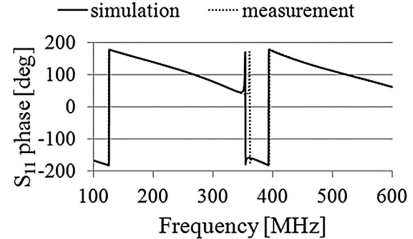

(b)

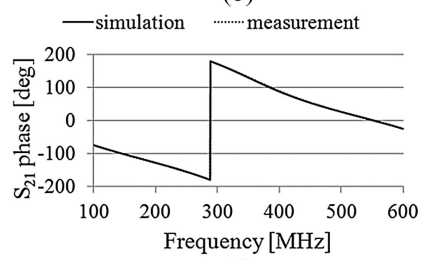

(d)

Fig. 5. Results for the coaxial container filled with distilled water: (a) magnitude of $S_{11}\left(\left|S_{11}\right|\right)$, (b) phase of $S_{11}$ (wrapped), (c) magnitude of $S_{21}\left(\left|S_{21}\right|\right)$, and (d) phase of $S_{21}$ (wrapped).

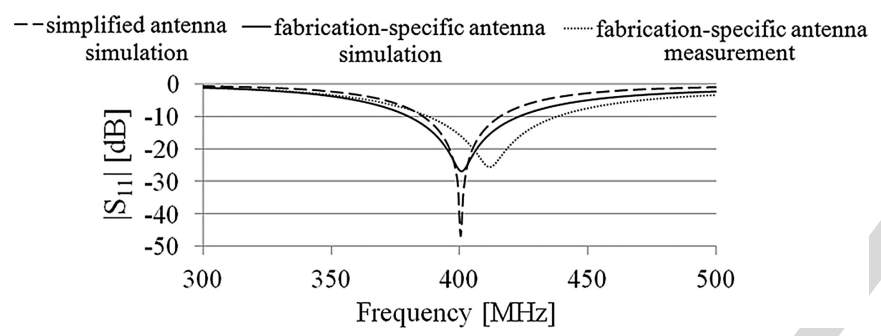

Fig. 6. Simulated and measured reflection coefficient frequency response of the simplified and fabrication-specific antennas.

the ground plane and the lower patch, the upper substrate contains the upper patch, and the superstrate has no metallization. Sprayable glue $3 \mathrm{M} 77$ is used to bond the three layers $\left(\varepsilon_{r}=2\right)$, which is found to exhibit an average thickness of $0.3 \mathrm{~mm}$ for the specific fabrication process. The antenna is fed through a 50-mmlong EZ-47 semirigid coaxial cable.

\section{B. Validation}

1) Fabrication-Specific Antenna: Metallization $\left(h_{m}\right)$, gluing $\left(\varepsilon_{r g}, h_{g}\right)$, and feeding (coaxial type, $L$ ) variables are set to the values imposed by the available fabrication approach, while the $R_{p}$ and $x_{i j}$ variables are optimized accordingly. Parameter values shown in Table II under "fabrication-specific" are found to tune the fabrication-specific antenna at $402 \mathrm{MHz}$ with a wide $10-\mathrm{dB}$ bandwidth (defined at $\left|S_{11}\right|<-10 \mathrm{~dB}$ ) of $44 \mathrm{MHz}$ covering the MICS band. Radii of the patches and meander lengths are significantly increased as compared to the simplified antenna. The aim is to counteract the increase in resonance frequency imposed by the low-permittivity glue layers. The simulated reflection coefficient frequency responses of the simplified and fabrication-specific antennas are shown in Fig. 6.

2) Sensitivity Tests: Sensitivity test results related to antenna design and experimental phantom uncertainties are indicated in Figs. 7 and 8, respectively. Only the antenna or phantom parameter under investigation is considered variable in each case, while all other parameters are kept constant and equal to those of the fabrication-specific antenna design (see Table II, fabricationspecific) and of the theoretical tissue model [see Fig. 1(a)]. (a)

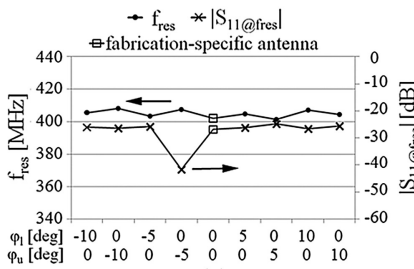

(c)

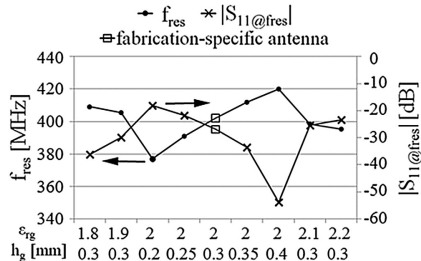

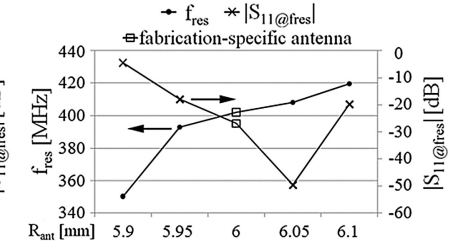

(b)

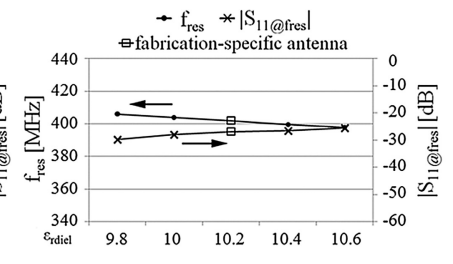

(d)
Fig. 7. Sensitivity test results $\left(f_{\text {res }},\left|S_{11 @ \text { fres }}\right|\right)$ related to prototype antenna parameters: (a) gluing $\left(\varepsilon_{r g}, h_{g}\right)$, (b) antenna radius $R_{\text {ant }}$, (c) rotation of the lower $\varphi_{l}$ and upper $\varphi_{u}$ patches, and (d) permittivity of the Rogers 3210 dielectric material, $\varepsilon_{r \text { diel }}$.

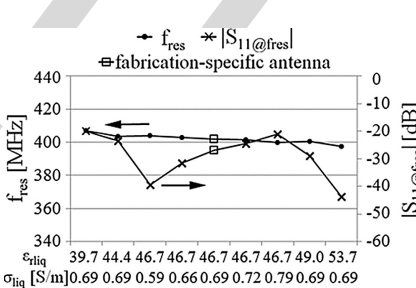

(a)

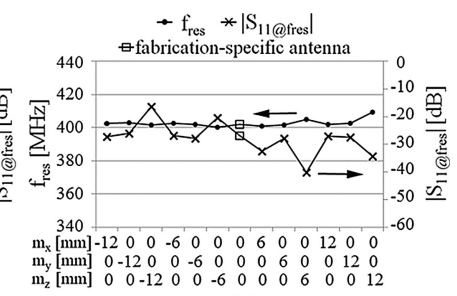

(b)
Fig. 8. Sensitivity test results $\left(f_{\text {res }},\left|S_{11 @ \text { fres }}\right|\right)$ related to phantom parameters: (a) permittivity $\varepsilon_{r \text { liq }}$ and conductivity $\sigma_{\text {liq }}$ of the skin-emulating liquid and (b) relative shift of the phantom from its original position $\left(m_{x}, m_{y}, m_{z}\right)$.

Resonance characteristics including the resonance frequency $f_{\text {res }}$ and $\left|S_{11 @ \text { fres }}\right|$ are recorded, while the performance of the fabrication-specific antenna is also shown for reference. Given the fabrication approach described in Section III-A, the following parameters are identified as potential sources of experimental uncertainties, and examined.

1) Gluing $\left(\varepsilon_{r g}, h_{g}\right)$ [see Fig. $\left.7(a)\right]$. Air bubbles accumulating within the glue prevent $\varepsilon_{r g}$ from being accurately determined. Furthermore, the adopted layer bonding process does not allow fine control of $h_{g}$. This is impaired not only by the glue itself, but also by the slight bump of the microsolder near the coaxial cable and the shorting pin that prevents perfect contact between the layers. Deviations of $\pm 10 \%$ and $33 \%$ in $\varepsilon_{r g}$ and $h_{g}$ are found to cause frequency detunings by up to $1.7 \%$ and $6.2 \%$, respectively;

2) Antenna radius $R_{\text {ant }}$ [see Fig. 7(b)]. Rogers 3210 requires significant mechanical stress (vertical pressure and torsion) for detaching the excess alignment material, thus degrading accuracy of the cutting procedure. A $0.2-\mathrm{mm}$ increase in $R_{\text {ant }}$ detunes the antenna by $4.4 \%$, whereas a $0.1-\mathrm{mm}$ decrease brings the copper patch sheets in direct contact with the tissue, thus, significantly degrading antenna resonance;

3) Relative rotation between the patches (indicated by the 344 rotation of the lower $\varphi_{l}$ and upper $\varphi_{u}$ patches around 345 
the z-axis) [see Fig. 7(c)]. Even though alignment marks are included in the photolithography masks, the alignment setup is relatively relaxed with respect to angular misalignment of the layers. Misalignment by $10^{\circ}$ is found to cause a maximum frequency detuning of only $1.2 \%$, thus, proving to be of minor importance. This justifies our choice for a relatively flexible alignment approach, while indicating the potential of relaxing the complexity of the assembling setup in order to benefit the gluing process that has been shown to be very critical. Positive and negative rotation angles correspond to clockwise and counterclockwise rotation around the $z$-axis, respectively;

4) Permittivity of the Rogers 3210 dielectric material, $\varepsilon_{\mathrm{rdiel}}$ [see Fig. 7(d)]. The typical value of the Rogers 3210 permittivity is defined to be 10.2 at $10 \mathrm{GHz}$ under $23{ }^{\circ} \mathrm{C}$. Frequency and temperature variations may slightly affect $\varepsilon_{\text {rdiel }}$ and degrade antenna performance. However, sensitivity tests indicate minor effects in antenna resonance, variations of \pm 0.4 in $\varepsilon_{\text {rdiel }}$ may lead to frequency detunings by up to only $1 \%$;

5) Permittivity $\varepsilon_{\mathrm{rliq}}$ and conductivity $\sigma_{\mathrm{liq}}$ of the skinsimulating liquid [see Fig. 8(a)]. Time and room temperature may perturb the properties of the mixture from their nominal values. Changes in $\varepsilon_{r \text { liq }}$ and $\sigma_{\text {liq }}$ by $15 \%$ are found to degrade antenna resonance by up to $1.2 \%$ and $0.5 \%$, respectively;

6) Relative antenna-phantom position (indicated by the relative shift of the phantom $\left(m_{x}, m_{y}, m_{z}\right)$ from its original location) [see Fig. 8(b)]. Since the antenna is manually positioned inside the phantom, slight deviations from the immersion scenario of Fig. 1(b) may occur. However, numerical results indicate insensitivity to antenna positioning inside the phantom as long as it is surrounded by skin-tissue.

3) In Vitro Testing: A skin-tissue-simulating liquid at $402 \mathrm{MHz}$ is prepared (56.18\% sugar, $2.33 \%$ salt, and $41.48 \%$ distilled water [2]) and its electric properties are measured using the technique described in Section II-D. Numerical and measured results are shown in Fig. 9, indicating adequacy of the mixture for in vitro testing. Dispersive, frequency-dependent $\varepsilon_{r}$ and $\sigma$ values of the skin-simulating liquid are used in the simulations, as shown in Fig. 10(solid line) [2]. The $\varepsilon_{r}$ and $\sigma$ values of actual skin-tissue are also shown for reference (dotted line) [22].

The prototype antenna is further built [see Fig. 11(a)], connected to a network analyzer, and immersed inside the liquid [see Fig. 11(b)]. The measured reflection coefficient frequency response is shown in Fig. 6 (dotted line). Good agreement exists between numerical and experimental results. A slight resonance shift of $10 \mathrm{MHz}(2.5 \%)$ is observed, which lies within the uncertainty allowances imposed by the sensitivity tests. Nevertheless, both simulation and measurement have an $\left|S_{11}\right|<-10 \mathrm{~dB}$ bandwidth which includes the MICS band.

\section{Performance Inside an Anatomical Head Model}

The proposed antenna model is finally evaluated within the scalp-implantation scenario of Fig. 1(b). The simplified antenna

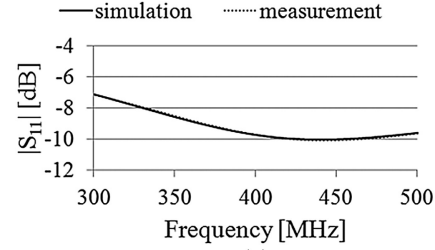

(a)

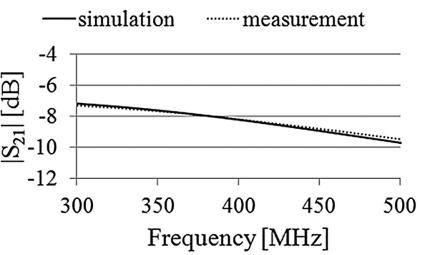

(c)

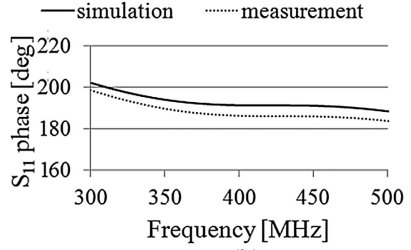

(b)

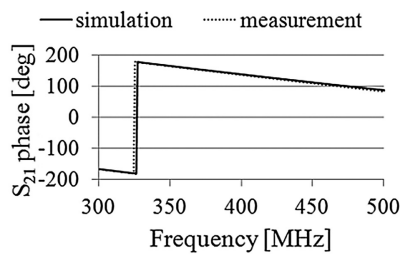

(d)
Fig. 9. Results for the coaxial container filled with skin-emulating liquid: (a) magnitude of $S_{11}\left(\left|S_{11}\right|\right)$, (b) phase of $S_{11}$ (wrapped), (c) magnitude of $S_{21}$ $\left(\left|S_{21}\right|\right)$, and (d) phase of $S_{21}$ (wrapped).

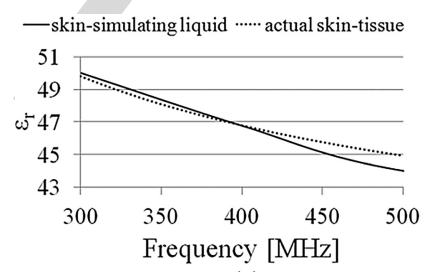

(a)

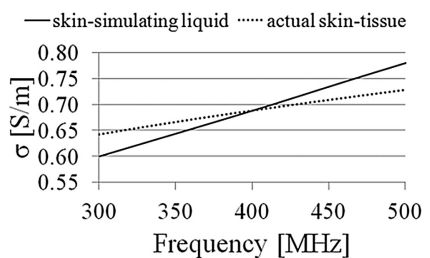

(b)
Fig. 10. Comparison of (a) permittivity $\varepsilon_{r}$ and (b) conductivity $\sigma$ of the skin-emulating liquid with the actual values for skin-tissue.

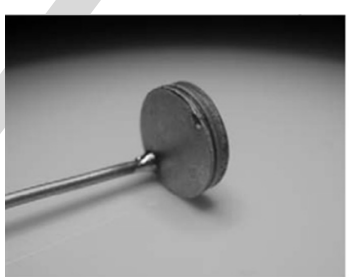

(a)

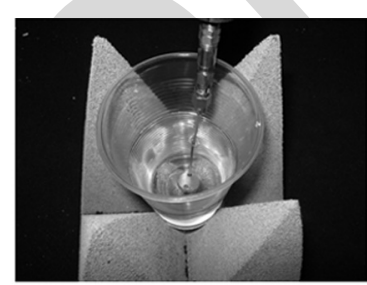

(b)
Fig. 11. (a) Fabricated prototype antenna and (b) in vitro testing setup.

is considered to provide generic results independent of the fab- 401 rication procedure, and fine-tuning is performed (see Table II 402 under "head-tuned") to achieve resonance in the MICS band 403 [see Fig. 12(a)]. Detuning issues for implantable antennas in- 404 side specific anatomical locations have been discussed by the 405 authors in [5], [17], and [26]. An infinitesimally thin wire and 406 a $50-\Omega$ voltage source model the $50-\Omega$ coaxial feed exciting 407 the antenna. The head-tuned antenna radiates an asymmetrical 408 far-field gain radiation pattern [see Fig. 12(b)], with a max- 409 imum gain of $-37.10 \mathrm{dBi}$ exhibited in the $(\theta, \varphi)=\left(110^{\circ}, 410\right.$ $90^{\circ}$ ) direction. Low-gain values are attributed to the small PIFA 411 size and high-tissue loss. Maximum 1-g-averaged (1-g-avg) and 412 10 -g- averaged (10-g-avg) specific absorption rate (SAR) val- 413 ues equal 324.74 and $65.09 \mathrm{~W} / \mathrm{kg}$, respectively, for a net in- 414 put power of $1 \mathrm{~W}$. The IEEE C95.1-1999 (1-g-avg SAR $\leq 415$ $1.6 \mathrm{~W} / \mathrm{kg})$ and C95.1-2005 (10-g-avg SAR $\leq 2 \mathrm{~W} / \mathrm{kg})$ safety 416 standards, thus, limit the maximum allowed net input power to 417 4.927 and $30.73 \mathrm{~mW}$, respectively [27]. Local SAR distribution 418 


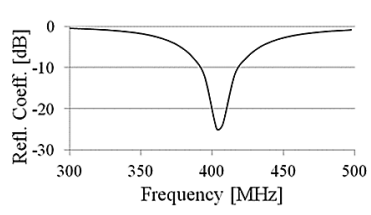

(a)

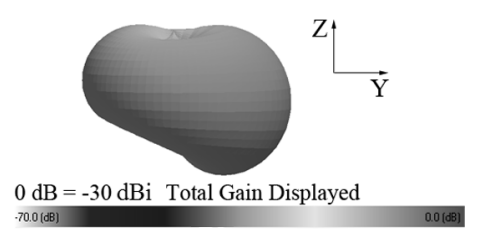

(b)

Fig. 12. (a) Reflection coefficient frequency response and (b) far-field gain radiation pattern of the head-tuned antenna implanted inside the anatomical head model [see Fig. 1(b)].

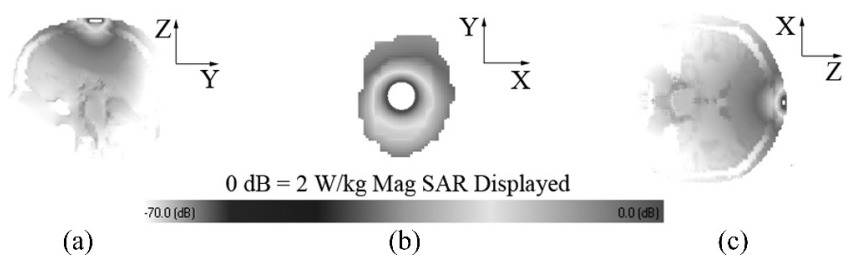

Fig. 13. Local SAR distribution in the (a) $y z$, (b) $x y$, and (c) $z x$ slices of the anatomical head model [see Fig. 1(b)] where maximum local SAR values have been calculated (net input power $=4.927 \mathrm{~mW}$ ).

considering a net-input power of $4.927 \mathrm{~mW}$ is shown in Fig. 13 for the slices where maximum local SAR values have been recorded.

\section{CONCLUSION}

We proposed a parametric model of a skin-implantable miniature PIFA for biotelemetry in the MICS band, and suggested a novel design-and-testing methodology for implantable antennas that incorporates gluing, metallization, and feeding considerations into numerical design. A low-cost, transmission-based technique was also described for reliably measuring the electric properties of liquids, without requiring specific commercial equipment.

Validation was further performed within a specific miniatureantenna-oriented fabrication approach. Sensitivity tests related to antenna design and phantom parameters indicated uncertainties of $0.5-6.2 \%$ in the exhibited resonance frequency, while relative antenna positioning was shown to be of minor significance. A resonance shift of $2.5 \%$ was observed in experimental testing as compared to simulations, which was within the expected uncertainty range.

Antenna implantation inside an anatomical head model required minor design modifications to refine tuning and exhibited an asymmetrical, low-gain (less than $-37.10 \mathrm{~dB}$ ) radiation pattern. Maximum net-input power levels of 4.927 and $30.73 \mathrm{~mW}$ were found to guarantee conformance with the IEEE C95.11999 and C95.1-2005 safety standards.

\section{ACKNOWLEDGMENT}

The authors would like to thank C. Brito and A. Almeida from Instituto Superior Técnico for prototype fabrication and measurement.

\section{REFERENCES}

[1] J. Kim and Y. Rahmat-Samii, "Implanted antennas inside a human body: Simulations, designs and characterizations," IEEE Trans. Microw. Theory Tech., vol. 52, no. 8, pp. 1934-1943, Aug. 2005.

[2] T. Karacolak, A. Z. Hood, and E. Topsakal, "Design of a dual-band implantable antenna and development of skin mimicking gels for continuous glucose monitoring," IEEE Trans. Microw. Theory Tech., vol. 56, no. 4 pp. 1001-1008, Apr. 2008.

[3] R. Warty, M.-R. Tofighi, U. Kawoos, and A. Rosen, "Characterization of implantable antennas for intracranial pressure monitoring: Reflection by and transmission through a scalp phantom," IEEE Trans. Microw. Theory Tech., vol. 56, no. 10, pp. 2366-2376, Oct. 2008.

[4] L. Xu and M. Q.-H. Meng, "Effects of dielectric parameters of human body on radiation characteristics of ingestible wireless device at operating frequency of $430 \mathrm{MHz}$," IEEE Trans. Biomed. Eng., vol. 56, no. 8 pp. 2083-2094, Aug. 2009.

[5] A. Kiourti, M. Christopoulou, and K. S. Nikita, "Performance of a novel miniature antenna implanted in the human head for wireless biotelemetry," in Proc. IEEE Int. Symp. Antennas Propag., Spokane, WA, 2011, pp. 392 395.

[6] "Medical implant communications service (MICS) federal register," Rules Reg., vol. 64, no. 240, pp. 69926-69934, Dec. 1999.

[7] "European Radiocommunications Commission (ERC) Recommendation 70-03 relating to the use of short range devices," presented at the Conf. Eur. Postal. Telecomm. Admin. (EPT), 1997, Paper CEPT/ERC 70-03 Annex 12.

[8] P. Soontornpipit, C. M. Furse, and Y. C. Chung, "Design of implantable microstrip antenna for communication with medical implants," IEEE Trans. Microw. Theory Tech., vol. 52, no. 8, pp. 1944-1951, Aug. 2004.

[9] P. Soontornpipit, C. M. Furse, and Y. C. Chung, "Miniaturized biocompatible microstrip antenna using genetic algorithm," IEEE Trans. Antennas Propag., vol. 53, no. 6, pp. 1939-1945, Jun. 2005.

[10] W.-C. Liu, F.-M. Yeh, and M. Ghavami, "Miniaturized implantable broadband antenna for biotelemetry communication," Microw. Opt. Technol. Lett., vol. 50, pp. 2407-2409, Sep. 2008.

[11] W.-C. Liu, S.-H. Chen, and C.-M. Wu, "Bandwidth enhancement and size reduction of an implantable PIFA antenna for biotelemetry devices," Microw. Opt. Technol. Lett., vol. 51, pp. 755-757, Mar. 2009.

[12] C.-M. Lee, T.-C. Yo, and C.-H. Luo, "Compact broadband stacked implantable antenna for biotelemetry with medical devices," in Proc. IEEE Annu. Wireless Microw. Technol. Conf., Dec. 4-5, 2006, pp. 1-4.

[13] A. Kiourti and K. S. Nikita, "Meandered versus spiral novel miniature PIFAs implanted in the human head: Tuning and performance," presented at the 2nd ICST Int. Conf. Wireless Mobile Commun. Healthcare, Kos Island, Greece, 2011, to be published.

[14] F. Merli and A. K. Skrivervik, "Design and measurement considerations for implantable antennas for telemetry applications," in Proc. 4th Europ Conf. Antennas Propag., Barcelona, Spain, Apr. 2010, pp. 1-5.

[15] F. Merli, L. Bolomey, J.-F Zurcher, G. Corradini, E. Meurville, and A. K. Skrivervik, "Design, Realization and measurements of a miniature antenna for implantable wireless communication systems," IEEE Trans. Antennas Propag., vol. 59, no. 10, pp. 3544-3555, Oct. 2011.

[16] W. Sun and Y.-X Yuan, Optimization theory and methods. New York: Springer-Verlag, 2006, ch. 5.

[17] A. Kiourti and K. S. Nikita, "Detuning issues and performance of a nove implantable antenna for telemetry applications," presented at the 6th Europ. Conf. Antennas Propag., Prague, Czech Republic, Mar. 2012.

[18] W. G. Scanlon and J. B. Burns, "Radiowave propagation from a tissueimplanted source at $418 \mathrm{MHz}$ and $916.5 \mathrm{MHz}$," IEEE Trans. Biomed Eng., vol. 47, no. 4, pp. 527-534, Apr. 2000.

[19] L. C. Chirwa, P. A. Hammond, S. Roy, and D. R. S. Cumming, "Electromagnetic radiation from ingested sources in the human intestine between $150 \mathrm{MHz}$ and $1.2 \mathrm{GHz}, "$ IEEE Trans. Biomed. Eng., vol. 50, no. 4 pp. 484-492, Apr. 2003.

[20] Ansoft High Frequency Structure Simulator (HFSS), Ver. 11, Ansoft Corporation, Pittsburgh, PA, 2008.

[21] XFDTD ${ }^{\circledR}$, Electromagnetic Solver Based on the Finite Difference Time Domain Method, Remcom, Inc., State College, PA.

[22] C. Gabriel, S. Gabriel, and E. Corthout, "The dielectric properties of biological tissues," Phys. Med. Biol., vol. 41, pp. 2231-2293, 1996.

[23] A. Kiourti, M. Tsakalakis, and K. S. Nikita, "Parametric study and design of implantable PIFAs for wireless biotelemetry," presented at the Proc. 2nd ICST Int. Conf. Wireless Mobile Commun. Healthcare, Kos Island, Greece, 2011 
[24] D. Popovic, L. McCartney, C. Beasley, M. Lazebnik, M. Okoniewsky, S. C. Hagness, and J. H. Booske, "Precision open-ended coaxial probes for in vivo and ex vivo dielectric spectroscopy of biological tissues at microwave frequencies," IEEE Trans. Microw. Theory Tech., vol. 53 , no. 5, pp. 1713-1722, May 2005.

[25] R. Zajicek, L. Oppl, and J. Vrbaf, "Broadband measurement of complex permittivity using reflection method and coaxial probes," Radioengineering, vol. 17, pp. 14-19, Apr. 2008.
[26] A. Kiourti and K. S. Nikita, "Miniature scalp-implantable antennas for telemetry in the MICS and ISM bands: Design, safety considerations and link budget analysis," IEEE Trans. Antennas Propag., to be published.

[27] IEEE Standard for Safety Levels with Respect to Human Exposure to 534 Radiofrequency Electromagnetic Fields, $3 \mathrm{kHz}$ to $300 \mathrm{GHz}$, IEEE Standard 535 C95.1-1999, 2005.

Authors' photographs and biographies not available at the time of publication. 537 
Q1. Author: Citation of "Fig.14" has been changed to "Fig. 13" in sentence "Local SAR ... been recorded." Please verify.

Q2. Author: Please provide name of the authors in Refs. [6], [7].

Q3. Author: Please provide the year information in Refs. [13], [21].

Q4. Author: Please update Ref. [26]. 